\title{
Detailed Kinetic Modeling of Iron Nanoparticle Synthesis from the Decomposition of $\mathrm{Fe}(\mathrm{CO})_{5}$
}

\section{John Z. Wen, C. Franklin Goldsmith, Robert W. Ashcraft, and William H. Green*}

Supporting Information Available: The exponents and contraction coefficients of Gaussian version $6-311+\mathrm{G}(\mathrm{d})$ basis for Fe, C and O; the DFT results including the multiplicities and Cartesian coordinates of ground states, the calculated vibrational frequencies, and zero-point energies; the thermodynamic data in CHEMKIN format and the mechanism for iron nanoparticle formation.

Part I: Gaussian version basis set: Standard basis: 6-311+G(d) (5D, 7F).

\begin{tabular}{|c|c|c|}
\hline \multicolumn{3}{|c|}{ Iron 0} \\
\hline \multirow[t]{7}{*}{ 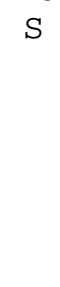 } & 61.00 & 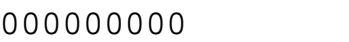 \\
\hline & $\odot .257539 \odot \odot \odot \odot D+\odot 6$ & $\odot .6141471350 \mathrm{D}-\odot 3$ \\
\hline & $\odot .386369 \odot \odot \odot \odot D+\odot 5$ & $\odot .4786112155 \mathrm{D}-\odot 2$ \\
\hline & $\odot .8891440 \odot \odot \odot D+\odot 4$ & $\odot .2439646550 \mathrm{D}-\odot 1$ \\
\hline & $\odot .2544 \odot 1 \odot \odot \odot \odot D+\odot 4$ & $\odot .9669640753 \mathrm{D}-01$ \\
\hline & $\odot .844777000 \odot \mathrm{D}+\odot 3$ & $\odot .2972260358 \mathrm{D}+\odot \odot$ \\
\hline & $\odot .31252700 \odot \odot D+\odot 3$ & $\odot .6653966545 \mathrm{D}+\odot \odot$ \\
\hline \multirow[t]{2}{*}{ S } & 11.00 & $\odot \odot \odot \odot \odot ० \odot ० \odot$ \\
\hline & $\odot .1255930000 \mathrm{D}+03$ & $\odot \odot \odot \odot \odot \odot \odot \odot D+\odot 1$ \\
\hline \multirow[t]{2}{*}{$\mathrm{s}$} & 11.00 & $\odot \odot \odot$ \\
\hline & $\odot .5349870 \odot \odot \odot D+\odot 2$ & $\odot .10 \odot \odot \odot \odot \odot \odot \odot \odot D+\odot 1$ \\
\hline \multirow[t]{2}{*}{$\mathrm{S}$} & 11.00 & 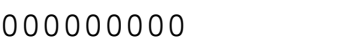 \\
\hline & $\odot .177151000 \odot \mathrm{D}+\odot 2$ & $\odot .100000000 \odot D+01$ \\
\hline \multirow[t]{2}{*}{$\mathrm{S}$} & 11.00 & $\odot \odot \odot \odot \odot \odot \odot \odot \odot ~$ \\
\hline & $\odot .7376770 \odot \odot \odot D+\odot 1$ & ๑๑९๑९८ \\
\hline \multirow[t]{2}{*}{$\mathrm{s}$} & 11.00 & $\odot \odot \odot$ \\
\hline & $\odot .201 \varepsilon$ & $\odot \odot \odot \odot D+\odot 1$ \\
\hline \multirow[t]{2}{*}{$\mathrm{s}$} & 11.00 & $\odot \odot \odot \odot \odot \odot \odot \odot \odot ~$ \\
\hline & $\odot .779935 \odot \odot \odot \odot D+\odot \odot$ & $\odot .100 \odot \odot \odot \odot \odot \odot$ \\
\hline \multirow[t]{2}{*}{$\mathrm{s}$} & 11.00 & $000 \mathrm{c}$ \\
\hline & $\odot .1142200000 \mathrm{D}+\odot \odot$ & 0000 \\
\hline \multirow[t]{2}{*}{$\mathrm{S}$} & 11.00 & $\odot \odot \odot ६$ \\
\hline & $\mathrm{D}-\odot 1$ & -01 \\
\hline & 51.00 & $\odot \odot \odot \odot \odot \odot \odot \odot \odot$ \\
\hline & $\odot .167840 \odot \odot \odot \odot D+\odot 4$ & $\odot .34774902$ \\
\hline & $\odot \odot \odot D+\odot 3$ & 0.2 \\
\hline & OD+ +03 & $3 D+00$ \\
\hline & $\odot .49115800 \odot \odot D+\odot 2$ & 0.3 \\
\hline & $\odot .2 \odot 5 \odot 35 \odot \odot \odot \odot D+\odot 2$ & $\odot .5989522998 \mathrm{D}+\odot \odot$ \\
\hline \multirow[t]{2}{*}{$\mathrm{P}$} & 11.00 & 000000000 \\
\hline & $\odot \odot \odot D+\odot 1$ & ๑००००००० \\
\hline \multirow[t]{2}{*}{$\mathrm{P}$} & 11.00 & 000000000 \\
\hline & $\odot \odot D+\odot 1$ & P \\
\hline \multirow[t]{2}{*}{$P$} & 11.00 & $\odot \odot \odot \odot \odot ० ० ० \odot$ \\
\hline & $\odot .152175 \odot \odot \odot \odot D+\odot 1$ & $\odot .10 \odot \odot \odot \odot \odot \odot \odot \odot D+\odot 1$ \\
\hline \multirow[t]{2}{*}{$\mathrm{P}$} & 11.00 & 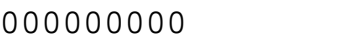 \\
\hline & $\odot .5926840 \odot \odot \odot D+\odot \odot$ & $\odot .100 \odot \odot \odot \odot \odot \odot \odot D+\odot$ \\
\hline \multirow[t]{3}{*}{ D } & 31.00 & 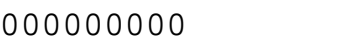 \\
\hline & $D+\odot 2$ & \\
\hline & $\odot .1154 \odot 3 \odot \odot \odot \odot D+\odot 2$ & $\odot .298419018$ \\
\hline
\end{tabular}




\begin{tabular}{|c|c|c|}
\hline & $\odot .3885430000 \mathrm{D}+01$ & 0.77359 \\
\hline & $\begin{array}{cc}11.0 \odot & 0.00 \odot \\
0.132380000 \odot \mathrm{D}+\odot 1\end{array}$ & $\begin{array}{l}000000000 \\
0.1000000000 \mathrm{D}+01\end{array}$ \\
\hline & 11.00 & $000 \mathrm{C}$ \\
\hline & $\odot .416680000 \odot D+\odot \odot$ & 0.10000000 \\
\hline & $\begin{array}{ll}11.00 & 0.000 \\
0 & 125670 \odot \odot \odot ค D-01\end{array}$ & ๑०००००००० \\
\hline & $\begin{array}{cc}11.00 & 0.00 \odot \\
0.134915000 \odot \mathrm{D}+\odot \odot\end{array}$ & $\begin{array}{l}000000 \odot \odot \odot \\
\odot .100 \odot \odot \odot \odot \odot \odot \odot D+01\end{array}$ \\
\hline & $\begin{array}{cc}11.00 & \odot .0 \odot \odot \\
0.41843000 \odot \odot \mathrm{D}-\odot 1\end{array}$ & $\begin{array}{l}000000 \odot \odot \odot \\
\odot .10 \odot \odot \odot \odot \odot \odot \odot \odot D+\odot 1\end{array}$ \\
\hline & $\begin{array}{cc}11.00 & 0.0 \odot \odot \\
\odot .113300 \odot \odot \odot \odot D+\odot \odot\end{array}$ & $\begin{array}{l}\odot \odot \odot \odot \odot \odot \odot \odot \odot ~ \\
\odot .10 \odot \odot \odot \odot \odot \odot \odot \odot D+\odot 1\end{array}$ \\
\hline & $\begin{array}{cc}11.0 \odot & \odot .0 \odot \odot) \\
0.105000 \odot \odot \odot \odot D+\odot 1\end{array}$ & $\begin{array}{l}\odot \odot \odot \odot \odot \odot \odot \odot \odot ~ \\
\odot .10000 \odot \odot \odot \odot \odot D+\odot\end{array}$ \\
\hline
\end{tabular}

\section{Carbon 0}

\begin{tabular}{|c|c|c|c|}
\hline & 61.00 & 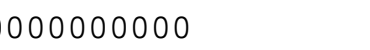 & \\
\hline & $\odot .4563240000 D+\odot 4$ & $\odot .1966650249 D-02$ & \\
\hline & $\odot .682024000 \odot \mathrm{D}+\odot 3$ & $\odot .1523060193 \mathrm{D}-01$ & \\
\hline & $\odot .15497300 \odot \odot D+\odot 3$ & $\odot .7612690966 \mathrm{D}-\odot 1$ & \\
\hline & $\odot .44455300 \odot \odot D+\odot 2$ & $\odot .2608010331 \mathrm{D}+\odot \odot$ & \\
\hline & $\odot .1302900000 \mathrm{D}+02$ & $\odot .6164620782 \mathrm{D}+\odot \odot$ & \\
\hline & $\odot .1827730000 \mathrm{D}+01$ & $\odot .2210060280 \mathrm{D}+\odot \odot$ & \\
\hline SP & 31.00 & 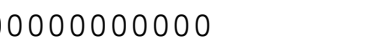 & \\
\hline & $\odot .20964200 \odot \odot D+\odot 2$ & $\odot .1146600807 \mathrm{D}+\odot \odot$ & $\odot .4024869267 \mathrm{D}-01$ \\
\hline & $\odot .48033100 \odot \odot D+01$ & $996477 D+\odot \odot$ & $+\odot \odot$ \\
\hline & $\odot .1459330000 \mathrm{D}+01$ & $-\odot .3030682134 \mathrm{D}-02$ & $0.8158538515 \mathrm{D}+00$ \\
\hline & 11.00 & 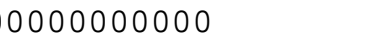 & \\
\hline & $\odot .483456 \odot \odot \odot \odot D+\odot \odot$ & $\odot .10 \odot \odot \odot \odot \odot \odot \odot \odot D+\odot 1$ & $0.10000 \odot 0 \odot \odot \odot D+01$ \\
\hline & 11.00 & ๑००००००००० & \\
\hline & $\odot .14558500 \odot \odot D+\odot \odot$ & $\odot .1000 \odot \odot \odot ० \odot ० D+01$ & $0.1000000000 \mathrm{D}+01$ \\
\hline & 11.00 & 0000000000 & \\
\hline & $\odot .4380000000 \mathrm{D}-01$ & $\odot .1000 \odot \odot \odot \odot \odot \odot D+01$ & $0.1000000000 \mathrm{D}+01$ \\
\hline & 11.00 & 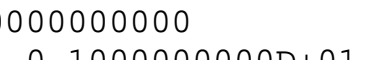 & \\
\hline
\end{tabular}

\section{Oxygen 0}
$\mathrm{S} \quad 61.00$
0.000000000000
$0.8588500000 D+04 \quad 0.1895150083 D-02$
$0.1297230000 \mathrm{D}+04 \quad 0.1438590063 \mathrm{D}-01$
$\odot .2992960000 \mathrm{D}+03 \quad 0.7073200310 \mathrm{D}-01$
$0.8737710000 \mathrm{D}+02 \quad 0.2400010105 \mathrm{D}+00$
$\odot .2567890000 \mathrm{D}+02 \quad 0.5947970261 \mathrm{D}+0 \odot$
$\odot .3740040000 \mathrm{D}+01 \quad 0.2808020123 \mathrm{D}+00$

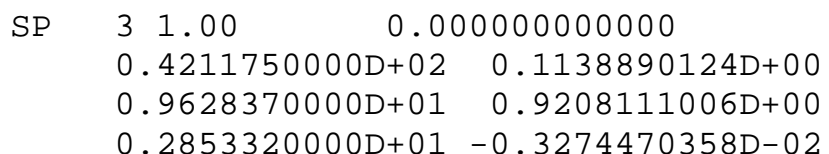
SP $\quad 11.00 \quad 0.000000000000$
$0.9056610000 \mathrm{D}+00 \quad 0.1000000000 \mathrm{D}+01 \quad 0.1000000000 \mathrm{D}+01$
SP $\quad 11.00 \quad 0.0000000000000$
$\odot .2556110000 \mathrm{D}+00 \quad 0.1000000000 \mathrm{D}+01 \quad 0.10000000000 \mathrm{D}+01$
SP $\quad 11.00 \quad 0.000000000000$
$0.8450000000 \mathrm{D}-01 \quad 0.100000000 \odot \mathrm{D}+01 \quad 0.100000000 \odot \mathrm{D}+01$
D $11.00 \quad 0.000000000000$
$\odot .3651139738 \mathrm{D}-01$
$\odot .2371529830 \mathrm{D}+\odot \odot$
$\odot .8197019412 \mathrm{D}+00$ 


\section{$\odot .1292000000 \mathrm{D}+01 \quad 0.1000000000 \mathrm{D}+01$}

\section{Part II: DFT Calculations of multiplicities and Ground States.}

\section{Fe3CO (Multiplicity: 9)}

Sum of electronic and zero-point energies: -3904.209879 (Hartree)

Orientation:

\begin{tabular}{cllccc} 
Center & Atomic & Atomic & \multicolumn{3}{c}{ Coordinates (Angstroms) } \\
Number & Number & Type & X & Y & $\mathrm{Z}$ \\
1 & 26 & 0 & 0.156489 & 0.301026 & 0.074612 \\
2 & 26 & 0 & 0.214691 & 0.265407 & 2.276979 \\
3 & 26 & 0 & 2.189245 & -0.535007 & 1.157543 \\
4 & 6 & 0 & -1.130663 & 1.292346 & 0.903234 \\
5 & 8 & 0 & -1.983589 & 2.019161 & 1.259811 \\
\hline
\end{tabular}

\section{Fe3CO (Multiplicity: 11) ground-state}

Sum of electronic and zero-point energies: -3904.221324 (Hartree)

Orientation:

\begin{tabular}{lllccc} 
Center & Atomic & Atomic & \multicolumn{3}{c}{ Coordinates (Angstroms) } \\
Number & Number & Type & $\mathrm{X}$ & $\mathrm{Y}$ & $\mathrm{Z}$ \\
1 & 26 & 0 & -0.027417 & -0.056156 & -0.061469 \\
2 & 26 & 0 & 0.043007 & -0.071736 & 2.253582 \\
3 & 26 & 0 & 2.125599 & 0.075090 & 1.156226 \\
4 & 6 & 0 & -1.579355 & 0.432687 & 0.845773 \\
5 & 8 & 0 & -2.654660 & 0.842629 & 1.037460
\end{tabular}

\section{Fe4CO (Multiplicity: 13)}

Sum of electronic and zero-point energies: -5167.882318 (Hartree)

Orientation:

\begin{tabular}{cllccc} 
Center & Atomic & Atomic & \multicolumn{3}{c}{ Coordinates (Angstroms) } \\
Number & Number & Type & X & Y & Z \\
1 & 26 & 0 & -0.010098 & -0.001859 & -0.008902 \\
2 & 26 & 0 & 0.013035 & 0.001273 & 2.546044 \\
3 & 26 & 0 & 1.989169 & 0.011726 & 1.260139 \\
4 & 26 & 0 & 0.354228 & -1.869408 & 1.387885 \\
5 & 6 & 0 & 0.339875 & -1.539420 & 3.503370 \\
6 & 8 & 0 & 0.612546 & -2.295106 & 4.355324
\end{tabular}

Fe4CO (Multiplicity: 15) ground-state

Sum of electronic and zero-point energies: -5167.897242 (Hartree) 
Orientation:

\begin{tabular}{lllccc} 
Center & Atomic & Atomic & \multicolumn{3}{c}{ Coordinates (Angstroms) } \\
Number & Number & Type & $\mathrm{X}$ & $\mathrm{Y}$ & $\mathrm{Z}$ \\
1 & 26 & 0 & -0.026533 & 0.149123 & -0.130498 \\
2 & 26 & 0 & -0.177065 & 0.007096 & 2.335755 \\
3 & 26 & 0 & 2.058760 & -0.154431 & 1.348784 \\
4 & 26 & 0 & 0.406231 & -1.873275 & 1.029100 \\
5 & 6 & 0 & -0.445421 & -1.664396 & 3.075513 \\
6 & 8 & 0 & -0.722433 & -2.532831 & 3.802943
\end{tabular}

Fe6CO (Multiplicity: 19)

Sum of electronic and zero-point energies: -7695.252880 (Hartree) Orientation:

\begin{tabular}{|c|c|c|c|c|c|}
\hline \multirow{2}{*}{$\begin{array}{l}\text { Center } \\
\text { Number }\end{array}$} & \multirow{2}{*}{$\begin{array}{l}\text { Atomic } \\
\text { Number }\end{array}$} & \multirow{2}{*}{$\begin{array}{l}\text { Atomic } \\
\text { Type }\end{array}$} & \multicolumn{3}{|c|}{ Coordinates (Angstroms) } \\
\hline & & & $\mathrm{X}$ & $\mathrm{Y}$ & $\mathrm{Z}$ \\
\hline 1 & 26 & 0 & 0.241713 & -0.153366 & 0.007578 \\
\hline 2 & 26 & 0 & -0.077733 & 0.013573 & 2.339754 \\
\hline 3 & 26 & 0 & 2.511574 & 0.053283 & -0.354415 \\
\hline 4 & 26 & 0 & 1.203454 & -1.897126 & 1.173361 \\
\hline 5 & 26 & 0 & 1.141778 & 1.829978 & 0.916242 \\
\hline 6 & 26 & 0 & 2.238135 & 0.050403 & 1.977182 \\
\hline 7 & 6 & 0 & 0.733005 & -1.420343 & -1.185913 \\
\hline 8 & 8 & 0 & 0.984139 & -2.227066 & -1.998162 \\
\hline
\end{tabular}

Fe6CO (Multiplicity: 21) ground-state

Sum of electronic and zero-point energies: -7695.253409 (Hartree)

Orientation:

\begin{tabular}{|c|c|c|c|c|c|}
\hline \multirow{2}{*}{$\begin{array}{l}\text { Center } \\
\text { Number }\end{array}$} & \multirow{2}{*}{$\begin{array}{l}\text { Atomic } \\
\text { Number }\end{array}$} & \multirow{2}{*}{$\begin{array}{l}\text { Atomic } \\
\text { Type }\end{array}$} & \multicolumn{3}{|c|}{ Coordinates (Angstroms) } \\
\hline & & & $\mathrm{X}$ & $\mathrm{Y}$ & $\mathrm{Z}$ \\
\hline 1 & 26 & 0 & -0.362139 & 0.545245 & 0.364095 \\
\hline 2 & 26 & 0 & -0.188799 & -0.090508 & 2.675965 \\
\hline 3 & 26 & 0 & 1.980902 & 0.203336 & 0.058945 \\
\hline 4 & 26 & 0 & 0.550025 & -1.742292 & 0.849415 \\
\hline 5 & 26 & 0 & 1.216850 & 1.746962 & 1.822966 \\
\hline 6 & 26 & 0 & 2.179432 & -0.447929 & 2.367761 \\
\hline 7 & 6 & 0 & 1.621310 & -2.889974 & -0.134989 \\
\hline 8 & 8 & 0 & 2.367684 & -3.512626 & -0.757707 \\
\hline
\end{tabular}

Fe7CO (Multiplicity: 23) ground-state

Sum of electronic and zero-point energies: -8958.927759 (Hartree) 
Orientation:

\begin{tabular}{lllccc} 
Center & Atomic & Atomic & \multicolumn{3}{c}{ Coordinates (Angstroms) } \\
Number & Number & Type & $\mathrm{X}$ & $\mathrm{Y}$ & $\mathrm{Z}$ \\
1 & 26 & 0 & 0.195685 & -0.038696 & -0.064741 \\
2 & 26 & 0 & -0.004360 & -0.057522 & 2.447193 \\
3 & 26 & 0 & 2.301174 & 0.167159 & 1.045241 \\
4 & 26 & 0 & 2.079441 & -1.068311 & 3.144691 \\
5 & 26 & 0 & 1.044198 & -1.864321 & 1.043541 \\
6 & 26 & 0 & 0.665849 & 1.966607 & 1.304701 \\
7 & 26 & 0 & 1.677511 & 1.321803 & 3.430607 \\
8 & 6 & 0 & 1.803432 & -1.078629 & -0.626127 \\
9 & 8 & 0 & 2.481064 & -1.337740 & -1.574293 \\
--------------------------------------------------------------------------
\end{tabular}

Harmonic frequencies $\left(\mathrm{cm}^{-1}\right)$ :

$\begin{array}{cccccc}1 & 2 & 3 & 4 & 5 & 6 \\ 69.3346 & 88.0723 & 101.4178 & 118.5790 & 119.8940 & 132.7225 \\ 7 & 8 & 9 & 10 & 11 & 12 \\ 136.0992 & 154.8145 & 160.9985 & 161.6975 & 177.2377 & 194.9631 \\ 13 & 14 & 15 & 16 & 17 & 18 \\ 215.4489 & 230.7586 & 253.8546 & 260.5602 & 269.1523 & 276.9468 \\ 19 & 20 & 21 & & & \\ 300.0737 & 396.9511 & 1748.2298 & & & \end{array}$

Fe7CO (Multiplicity: 25)

Sum of electronic and zero-point energies: -8958.896923 (Hartree)

Orientation:

\begin{tabular}{|c|c|c|c|c|c|}
\hline \multirow{2}{*}{$\begin{array}{l}\text { Center } \\
\text { Number }\end{array}$} & \multirow{2}{*}{$\begin{array}{l}\text { Atomic } \\
\text { Number }\end{array}$} & \multirow{2}{*}{$\begin{array}{l}\text { Atomic } \\
\text { Type }\end{array}$} & \multicolumn{3}{|c|}{ Coordinates (Angstroms) } \\
\hline & & & $X$ & $\mathrm{Y}$ & Z \\
\hline 1 & 26 & 0 & 0.101037 & 0.232102 & -0.283884 \\
\hline 2 & 26 & 0 & -0.021314 & -0.134595 & 2.435577 \\
\hline 3 & 26 & 0 & 2.278511 & 0.043396 & 0.880132 \\
\hline 4 & 26 & 0 & 1.968369 & -1.343793 & 3.066401 \\
\hline 5 & 26 & 0 & 0.924040 & -1.888768 & 0.924357 \\
\hline 6 & 26 & 0 & 0.709643 & 1.895707 & 1.423438 \\
\hline 7 & 26 & 0 & 2.097240 & 1.003119 & 3.236839 \\
\hline 8 & 6 & 0 & 3.557021 & -0.015300 & -0.561105 \\
\hline 9 & 8 & 0 & 4.261898 & -0.094184 & -1.459658 \\
\hline
\end{tabular}

Harmonic frequencies $\left(\mathrm{cm}^{-1}\right)$ :

$\begin{array}{cccccc}1 & 2 & 3 & 4 & 5 & 6 \\ 24.7037 & 29.9000 & 52.2925 & 71.0257 & 88.9912 & 100.1024\end{array}$


$\begin{array}{llllll}7 & 8 & 9 & 10 & 11 & 12\end{array}$

$\begin{array}{llllll}116.5724 & 131.7367 & 150.0678 & 157.3134 & 161.2981 & 192.4370\end{array}$

$\begin{array}{llllll}13 & 14 & 15 & 16 & 17 & 18\end{array}$

$\begin{array}{llllll}200.2475 & 205.7478 & 232.6014 & 245.9860 & 256.4626 & 312.5692\end{array}$

$\begin{array}{lll}19 & 20 & 21\end{array}$

$329.2116 \quad 348.8027 \quad 2036.9790$

\section{Fe2(CO)2 (Multiplicity: 5)}

Sum of electronic and zero-point energies: -2753.891935 (Hartree)

Orientation:

\begin{tabular}{lllccc} 
Center & Atomic & Atomic & \multicolumn{3}{c}{ Coordinates (Angstroms) } \\
Number & Number & Type & $\mathrm{X}$ & $\mathrm{Y}$ & $\mathrm{Z}$ \\
1 & 8 & 0 & -0.005401 & 0.000738 & 0.020193 \\
2 & 6 & 0 & 0.059989 & -0.001055 & 1.180529 \\
3 & 26 & 0 & 0.230802 & -0.003138 & 2.979877 \\
4 & 26 & 0 & -1.907924 & 0.041351 & 2.645495 \\
5 & 6 & 0 & -0.449441 & 0.015925 & 4.654244 \\
6 & 8 & 0 & -0.853680 & 0.028540 & 5.742903 \\
\hline
\end{tabular}

Harmonic frequencies $\left(\mathrm{cm}^{-1}\right)$ :

$\begin{array}{cccccc}1 & 2 & 3 & 4 & 5 & 6 \\ 27.6474 & 54.5553 & 94.6681 & 261.9124 & 312.1892 & 333.9239 \\ 7 & 8 & 9 & 10 & 11 & 12 \\ 448.2064 & 516.6890 & 520.1231 & 540.1649 & 1939.642 & 2005.4821 \\ - & - & -\end{array}$

\section{Fe2(CO)2 (Multiplicity: 7 \#1) ground-state}

Sum of electronic and zero-point energies: -2753.908262 (Hartree)

Orientation:

\begin{tabular}{lllccc} 
Center & Atomic & Atomic & \multicolumn{3}{c}{ Coordinates (Angstroms) } \\
Number & Number & Type & $\mathrm{X}$ & $\mathrm{Y}$ & $\mathrm{Z}$ \\
1 & 8 & 0 & 0.284507 & -0.072936 & 0.156055 \\
2 & 6 & 0 & 0.121347 & -0.014149 & 1.293407 \\
3 & 26 & 0 & -0.183017 & 0.081943 & 3.096331 \\
4 & 26 & 0 & -2.358082 & 0.617928 & 2.520612 \\
5 & 6 & 0 & -0.744672 & 0.232575 & 4.832507 \\
6 & 8 & 0 & -1.123526 & 0.320067 & 5.915255 \\
-
\end{tabular}

Harmonic frequencies $\left(\mathrm{cm}^{-1}\right)$ :

\begin{tabular}{|c|c|c|c|c|c|}
\hline $\begin{array}{c}1 \\
69.0156\end{array}$ & $\begin{array}{c}2 \\
71.8956\end{array}$ & $\begin{array}{c}3 \\
85.5698\end{array}$ & $\begin{array}{c}4 \\
239.0301\end{array}$ & $\begin{array}{c}5 \\
335.4109\end{array}$ & $\begin{array}{c}6 \\
390.9195\end{array}$ \\
\hline
\end{tabular}




\section{Fe2(CO)2 (Multiplicity: 7 \#2) linear}

Sum of electronic and zero-point energies: -2753.895838 (Hartree)

Orientation:

\begin{tabular}{|c|c|c|c|c|c|}
\hline Center & Atomic & Atomic & Coord & dinates (Angs & troms) \\
\hline Number & Number & Type & $\mathrm{X}$ & $\mathrm{Y}$ & $\mathrm{Z}$ \\
\hline 1 & 8 & 0 & 0.103653 & -0.023415 & -0.163693 \\
\hline 2 & 6 & 0 & 0.063560 & -0.011023 & 0.982943 \\
\hline 3 & 26 & 0 & 0.032267 & 0.002968 & 2.839031 \\
\hline 4 & 26 & 0 & 0.212061 & -0.019690 & 5.431140 \\
\hline 5 & 6 & 0 & 0.181791 & -0.003941 & 7.287575 \\
\hline 6 & 8 & 0 & 0.142639 & 0.010158 & 8.434112 \\
\hline Harmonic & frequencies & $\mathrm{s}\left(\mathrm{cm}^{-1}\right)$ : & & & \\
\hline 1 & 2 & 3 & 4 & 5 & 6 \\
\hline 25.1954 & 26.1496 & 30.0509 & 172.3255 & 280.2493 & 291.3837 \\
\hline 7 & 8 & 9 & 10 & 11 & 12 \\
\hline 298.0389 & ר 298.4107 & 434.5851 & 444.6869 & 2063.108 & 2089.0398 \\
\hline
\end{tabular}

\section{Fe2(CO)3 (Multiplicity: 5 \#1) ground-state}

Sum of electronic and zero-point energies: -2867.235454 (Hartree)

Orientation:

\begin{tabular}{lllclc} 
Center & Atomic & Atomic & \multicolumn{3}{c}{ Coordinates (Angstroms) } \\
Number & Number & Type & $\mathrm{X}$ & $\mathrm{Y}$ & $\mathrm{Z}$ \\
1 & 26 & 0 & 0.025933 & -0.027332 & -0.058294 \\
2 & 26 & 0 & 0.189444 & 0.411247 & 2.176786 \\
3 & 6 & 0 & 1.830480 & -0.032015 & 0.092594 \\
4 & 6 & 0 & -1.597667 & 0.237423 & 0.663928 \\
5 & 6 & 0 & 1.044173 & -0.338761 & 3.607653 \\
6 & 8 & 0 & 2.979144 & -0.011106 & 0.225234 \\
7 & 8 & 0 & -2.655846 & 0.401964 & 1.128238 \\
8 & 8 & 0 & 1.616532 & -0.895215 & 4.431686
\end{tabular}

Harmonic frequencies $\left(\mathrm{cm}^{-1}\right)$ :

$\begin{array}{cccccc}1 & 2 & 3 & 4 & 5 & 6 \\ 40.3788 & 54.6632 & 65.2656 & 89.6588 & 98.3648 & 232.4825 \\ 7 & 8 & 9 & 10 & 11 & 12 \\ 331.1255 & 340.3999 & 356.1901 & 391.0211 & 451.7717 & 453.5822\end{array}$


$\begin{array}{llllll}13 & 14 & 15 & 16 & 17 & 18\end{array}$ $\begin{array}{llllll}509.0117 & 530.1701 & 543.8998 & 1926.668 & 2005.167 & 2075.0339\end{array}$

\section{Fe2(CO)3 (Multiplicity: 5 \#2)}

Sum of electronic and zero-point energies: -2867.220720 (Hartree)

Orientation:

\begin{tabular}{|c|c|c|c|c|c|}
\hline Center & Atomic & Atomic & Coorc & linates (Angs & stroms) \\
\hline Number I & Number & Type & $X$ & Y & Z \\
\hline 1 & 26 & 0 & -0.036743 & -0.185333 & -0.037266 \\
\hline 2 & 26 & 0 & -0.192775 & 0.024563 & 2.332820 \\
\hline 3 & 6 & 0 & 0.726126 & 0.082195 & -1.683917 \\
\hline 4 & 6 & 0 & 0.601590 & 1.276607 & 0.755702 \\
\hline 5 & 6 & 0 & -1.109233 & -0.837312 & 3.631066 \\
\hline 6 & 8 & 0 & 1.306760 & 0.331603 & -2.639535 \\
\hline 7 & 8 & 0 & 1.028174 & 2.356800 & 0.905463 \\
\hline 8 & 8 & 0 & -1.733362 & -1.451873 & 4.375605 \\
\hline Harmonic $\mathrm{f}$ & frequencies & $\mathrm{s}\left(\mathrm{cm}^{-1}\right)$ : & & & \\
\hline 1 & 2 & 3 & 4 & 5 & 6 \\
\hline 27.5753 & 45.6130 & 47.0307 & 70.1234 & 158.6380 & 202.7138 \\
\hline 7 & 8 & 9 & 10 & 11 & 12 \\
\hline 306.9390 & 339.2366 & 343.7993 & 383.5079 & 396.1030 & 442.5875 \\
\hline 13 & 14 & 15 & 16 & 17 & 18 \\
\hline 462.8698 & $\begin{array}{l}3 \\
489.4230\end{array}$ & 517.3897 & 1895.133 & 2034.528 & 2081.630 \\
\hline
\end{tabular}

Fe2(CO)3 (Multiplicity: 5 \#3)

Sum of electronic and zero-point energies: -2867.227175 (Hartree)

Orientation:

\begin{tabular}{cllcll}
\hline Center & Atomic & Atomic & \multicolumn{3}{c}{ Coordinates (Angstroms) } \\
Number & Number & Type & \multicolumn{1}{c}{$\mathrm{X}$} & $\mathrm{Y}$ & $\mathrm{Z}$ \\
1 & 26 & 0 & 0.040005 & -0.118520 & 0.157360 \\
2 & 26 & 0 & -0.019813 & 0.110588 & 2.441523 \\
3 & 6 & 0 & 1.835918 & -0.007193 & -0.075312 \\
4 & 6 & 0 & -0.417200 & 1.489874 & 1.118581 \\
5 & 6 & 0 & 0.282140 & -1.461930 & 3.284942 \\
6 & 8 & 0 & 2.979137 & 0.046367 & -0.188213 \\
7 & 8 & 0 & -0.726494 & 2.580368 & 0.803664 \\
8 & 8 & 0 & 0.456774 & -2.496022 & 3.758652
\end{tabular}

Harmonic frequencies $\left(\mathrm{cm}^{-1}\right)$ :

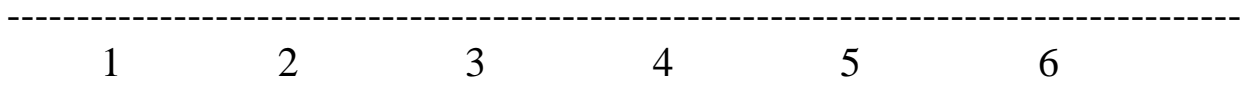


$\begin{array}{llllll}39.7761 & 54.9544 & 75.6184 & 83.5545 & 172.7074 & 236.4063\end{array}$

$\begin{array}{llllll}7 & 8 & 9 & 10 & 11 & 12\end{array}$

$288.5333 \quad 317.6598 \quad 341.3440 \quad 392.7557 \quad 400.5599 \quad 456.4206$

$\begin{array}{llllll}13 & 14 & 15 & 16 & 17 & 18\end{array}$

$\begin{array}{llllll}468.4601 & 497.3628 & 510.9665 & 1865.395 & 2018.199 & 2067.2899\end{array}$

Fe2(CO)3 (Multiplicity: 7)

Sum of electronic and zero-point energies: -2867.222154 (Hartree)

Orientation:

\begin{tabular}{|c|c|c|c|c|c|}
\hline \multirow{2}{*}{$\begin{array}{l}\text { Center } \\
\text { Number }\end{array}$} & \multirow{2}{*}{$\begin{array}{l}\text { Atomic } \\
\text { Number }\end{array}$} & \multirow{2}{*}{$\begin{array}{l}\text { Atomic } \\
\text { Type }\end{array}$} & \multicolumn{3}{|c|}{ Coordinates (Angstroms) } \\
\hline & & & $\mathrm{X}$ & $\mathrm{Y}$ & $\mathrm{Z}$ \\
\hline 1 & 26 & 0 & 0.125690 & 0.329576 & -0.231214 \\
\hline 2 & 26 & 0 & 0.535366 & 0.203597 & 2.066727 \\
\hline 3 & 6 & 0 & 1.844516 & -0.261262 & 0.162615 \\
\hline 4 & 6 & 0 & -0.638655 & 1.587591 & 1.000508 \\
\hline 5 & 6 & 0 & 0.484100 & -1.314936 & 3.120176 \\
\hline 6 & 8 & 0 & 2.936840 & $-0.64327 €$ & 0.154044 \\
\hline 7 & 8 & 0 & -1.298993 & 2.509058 & 1.276255 \\
\hline 8 & 8 & 0 & 0.441603 & $-2.26681 \epsilon$ & 3.752085 \\
\hline \multicolumn{6}{|c|}{ Harmonic frequencies $\left(\mathrm{cm}^{-1}\right)$ : } \\
\hline 1 & 2 & 3 & 4 & 5 & 6 \\
\hline 24.9445 & 50.8305 & 67.5360 & 106.1611 & 177.0709 & 188.6052 \\
\hline 7 & 8 & 9 & 10 & 11 & 12 \\
\hline 260.6987 & $7 \quad 306.2989$ & 327.1889 & 341.3390 & 384.7009 & 404.0717 \\
\hline 13 & 14 & 15 & 16 & 17 & 18 \\
\hline 407.8059 & 439.9949 & 449.3125 & 1910.259 & 1983.284 & 2087.873 \\
\hline
\end{tabular}

Fe3(CO)2 (Multiplicity: 11 \#1) ground-state

Sum of electronic and zero-point energies: -4017.549108 (Hartree)

Orientation:

\begin{tabular}{|c|c|c|c|c|c|}
\hline Center & Atomic & Atomic & \multicolumn{3}{|c|}{ Coordinates (Angstroms) } \\
\hline Number & Number & Type & $\mathrm{X}$ & $\mathrm{Y}$ & $\mathrm{Z}$ \\
\hline 1 & 26 & 0 & -0.345465 & 0.014099 & 0.094429 \\
\hline 2 & 26 & 0 & 0.095535 & -0.003835 & 2.558953 \\
\hline 3 & 26 & 0 & 2.036297 & -0.017864 & 0.873441 \\
\hline 4 & 6 & 0 & -1.055259 & 0.000894 & 4.006949 \\
\hline 5 & 6 & 0 & 2.333358 & -0.009294 & -0.952488 \\
\hline 6 & 8 & 0 & -1.791834 & 0.003886 & 4.886558 \\
\hline 7 & 8 & 0 & 2.577738 & -0.001502 & -2.078526 \\
\hline
\end{tabular}


Harmonic frequencies $\left(\mathrm{cm}^{-1}\right)$ :

$\begin{array}{cccccc}1 & 2 & 3 & 4 & 5 & 6 \\ 34.6829 & 39.5731 & 62.4388 & 63.4802 & 133.3643 & 165.4076 \\ 7 & 8 & 9 & 10 & 11 & 12 \\ 207.6509 & 302.9364 & 336.3062 & 338.6789 & 382.6250 & 430.8338 \\ 13 & 14 & 15 & & & \\ 439.0040 & 2017.145 & 2060.759 & & & \end{array}$

Fe3(CO)2 (Multiplicity: 11 \#2) linear

Sum of electronic and zero-point energies: -4017.509729 (Hartree)

Orientation:

\begin{tabular}{lllccc} 
Center & Atomic & Atomic & \multicolumn{3}{c}{ Coordinates (Angstroms) } \\
Number & Number & Type & X & Y & $\mathrm{Z}$ \\
1 & 6 & 0 & 0.000000 & 0.000000 & 0.000000 \\
2 & 26 & 0 & 0.000000 & 0.000000 & 1.856856 \\
3 & 26 & 0 & -0.000111 & 0.000000 & 4.404467 \\
4 & 26 & 0 & -0.000223 & 0.000000 & 6.952078 \\
5 & 6 & 0 & -0.000304 & 0.000000 & 8.808934 \\
6 & 8 & 0 & -0.000354 & 0.000000 & 9.955671 \\
7 & 8 & 0 & 0.000025 & 0.000000 & -1.146737
\end{tabular}

Harmonic frequencies $\left(\mathrm{cm}^{-1}\right)$ :

$\begin{array}{cccccc}1 & 2 & 3 & 4 & 5 & 6 \\ -13.5290 & 51.7303 & 64.8898 & 70.1522 & 75.5051 & 112.6257 \\ 7 & 8 & 9 & 10 & 11 & 12 \\ 185.1125 & 312.5868 & 317.1858 & 431.3313 & 442.7612 & 590.4751 \\ 13 & 14 & 15 & & & \\ 609.4354 & 2060.823 & 2083.738 & & & \end{array}$

\section{Part III: Thermo Data in CHEMIKIN Format.}

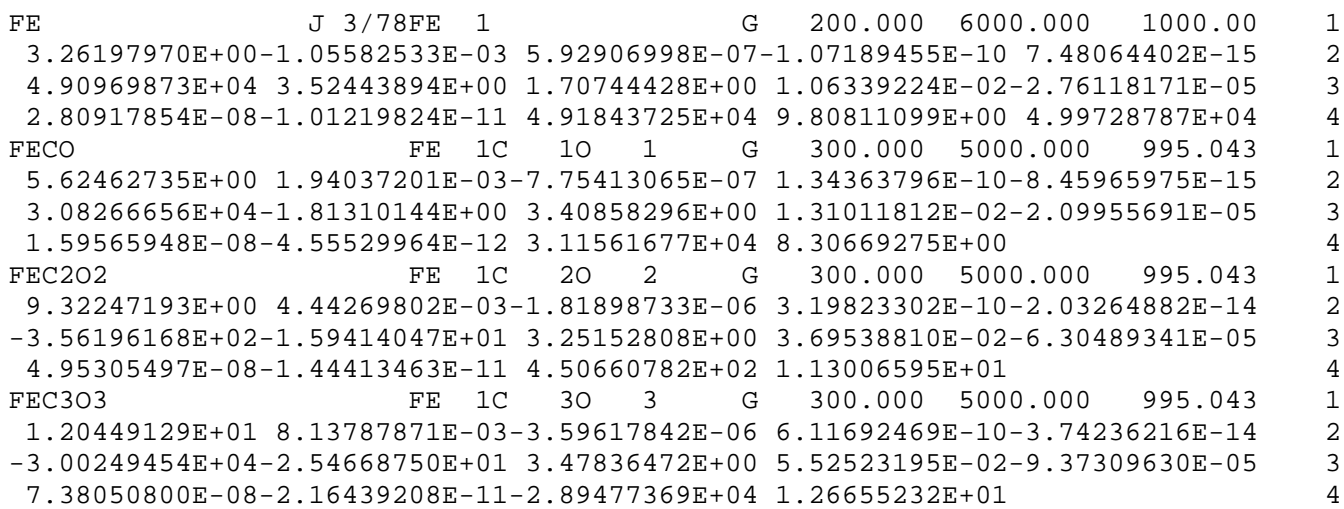


$\begin{array}{lllllllll}\text { FEC404 FE } & 1 C & 40 & 4 & \text { G } & 300.000 & 5000.000 & 995.043\end{array}$

$1.55310513 \mathrm{E}+01$ 1.10282893E- $02-4.85729219 \mathrm{E}-06 \quad 8.27911916 \mathrm{E}-10-5.07710499 \mathrm{E}-14$

$-5.98957193 E+04-3.81826380 E+01 \quad 3.26991724 E+0 \odot \quad 7.88840708 E-02-1.35137155 E-04$

$1.06866217 \mathrm{E}-07-3.14038635 \mathrm{E}-11-5.83748130 \mathrm{E}+04$ 1.62905969E+01

$\begin{array}{lllllllll}\text { FEC505 } & \text { FE } & 1 C & 50 & 5 & \text { G } & 300.000 & 5000.000 & 995.043\end{array}$

1.91981304E+01 1.33772655E- $02-5.76895491 E-06$ 9.69674659E-10-5.88650476E-14

$-9.39736166 \mathrm{E}+04-6.01402410 \mathrm{E}+012.95096214 \mathrm{E}+0 \odot \quad 9.32352266 \mathrm{E}-02-1.48079642 \mathrm{E}-04$

$1.11006929 \mathrm{E}-07-3.13962275 \mathrm{E}-11-9.14603744 \mathrm{E}+04$ 1.45438593E+01

$\begin{array}{llllllll}\text { FE2 } & \text { FE } & 20 & 0 & \text { G } & 300.000 & 5000.000 & 995.043\end{array}$

4. $41856135 \mathrm{E}+00 \quad 6.26250187 \mathrm{E}-05-1.37435667 \mathrm{E}-08$ 1.30310335E-12-4.24314284E-17

$8.48734565 \mathrm{E}+\odot 4 \quad 5.46918512 \mathrm{E}+\odot \odot \quad 3.43223456 \mathrm{E}+\odot \odot \quad 4.01763676 \mathrm{E}-\odot 3-5.96081618 \mathrm{E}-\odot 6$

3.97572003E-09-9.96071694E-13 8.50702366E+@4 1.02252155E+@1

$\begin{array}{lllllll}\text { FE3 } & \text { FE } 30 & 0 & \text { G } & 300.000 & 5000.000 & 995.043\end{array}$

$6.13878410 \mathrm{E}+0 \odot \quad 1.47952037 \mathrm{E}-03-8.07056631 \mathrm{E}-07 \quad 1.42094505 \mathrm{E}-10-8.76980691 \mathrm{E}-15$

$1.12284537 \mathrm{E}+05 \quad 6.14606737 \mathrm{E}+00 \quad 3.85098463 \mathrm{E}+00$ 1.77571692E-02-3.60193758E- 05

$3.08855126 \mathrm{E}-08-9.52971898 \mathrm{E}-12 \quad 1.12389287 \mathrm{E}+05$ 1.54105683E+01

$\begin{array}{llllllll}\text { FE4 } & \text { FE } & 40 & 0 & \text { G } & 300.000 & 5000.000 & 995.043\end{array}$

8.31853111E+@९ 2.83954689E-03-1.54153876E- $06 \quad 2.82452578 E-10-1.80432463 E-14$

$1.36348307 \mathrm{E}+05-1.60593260 \mathrm{E}-01 \quad 3.79578936 \mathrm{E}+0 \odot \quad 3.76654735 \mathrm{E}-02-7.91323111 \mathrm{E}-05$

6.90784261E-08-2.15264313E-11 1.36424361E+05 1.74959654E+01

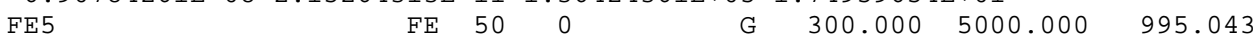

1. $06000155 \mathrm{E}+01$ 4.00513661E-03-2.16172017E-06 3.99283665E-10-2.56895161E-14

$1.57809949 \mathrm{E}+05-9.47400170 \mathrm{E}+0 \odot \quad 3.70695410 \mathrm{E}+\odot \odot \quad 5.64456239 \mathrm{E}-02-1.18495462 \mathrm{E}-\odot 4$

$1.03319395 \mathrm{E}-07-3.21594886 \mathrm{E}-11$ 1.57957412E+05 1.75947091E+01

$\begin{array}{lllllll}\text { FE6 } & \text { FE } 60 & 0 & \text { G } & 300.000 & 5000.000 & 995.043\end{array}$

1.30447460E+01 4.83071436E-03-2.57689911E-06 4.73481934E-10-3.03688204E-14

$1.73235349 \mathrm{E}+\Theta 5-1.95103804 \mathrm{E}+01 \quad 3.55612687 \mathrm{E}+\odot \odot \quad 7.21384632 \mathrm{E}-02-1.48005724 \mathrm{E}-\odot 4$

$1.27364539 \mathrm{E}-07-3.93117338 \mathrm{E}-11$ 1.73679878E+05 $1.89646648 \mathrm{E}+01$

$\begin{array}{llllllll}\text { FE7 } & \text { FE } & 70 & \odot & \text { G } & 300.000 & 5000.000 & 995.043\end{array}$

$1.51176988 \mathrm{E}+01 \quad 6.41461339 \mathrm{E}-03-3.44979066 \mathrm{E}-06 \quad 6.42883841 \mathrm{E}-10-4.16722023 \mathrm{E}-14$

$1.91942170 \mathrm{E}+05-2.41674611 \mathrm{E}+01 \quad 3.54388356 \mathrm{E}+\odot \odot \quad 9.41451303 \mathrm{E}-02-1.97816007 \mathrm{E}-\odot 4$

$1.72482102 \mathrm{E}-07-5.36713798 \mathrm{E}-11$ 1.92205603E+05 2.13619017E+@1

$\begin{array}{llllllll}\text { B01 } & \text { FE } 80 & 0 & \text { S } & 300.000 & 5000.000 & 995.043\end{array}$

$1.76789203 \mathrm{E}+01 \quad 7.03784191 \mathrm{E}-03-3.75347613 \mathrm{E}-06 \quad 6.94663767 \mathrm{E}-10-4.48181344 \mathrm{E}-14$

$2.14251258 \mathrm{E}+05-4.27140202 \mathrm{E}+01 \quad 3.36156287 \mathrm{E}+0 \odot \quad 1.08734360 \mathrm{E}-01-2.23600750 \mathrm{E}-04$

1.92572381E-07-5.94544274E-11 2.14915273E+05 1.53070635E+01

$\begin{array}{lllllllll}\text { FE2CO } & \text { FE } & 2 C & 10 & 1 & \text { G } & 300.000 & 5000.000 & 995.043\end{array}$

$7.57186001 \mathrm{E}+0 \odot \quad 2.78532572 \mathrm{E}-03-1.21561329 \mathrm{E}-\odot 6 \quad 2.23595507 \mathrm{E}-10-1.46306070 \mathrm{E}-14$

$5.60809401 E+04-2.54133724 E+0 \odot \quad 3.37935345 E+0 \odot \quad 2.93465021 E-02-5.58898148 E-05$

4.66592206E- -8-1.41447252E-11 5.64347050E+@4 1.52493784E+@1

$\begin{array}{llllllll}\text { FE3CO } & \text { FE } 3 C & 10 & 1 & \text { G } & 300.000 & 5000.000 & 995.043\end{array}$

$9.54951314 \mathrm{E}+0 \odot \quad 4.78883568 \mathrm{E}-03-2.36221848 \mathrm{E}-06 \quad 4.22033065 \mathrm{E}-10-2.66280897 \mathrm{E}-14$

$8.44487353 \mathrm{E}+04-7.62769590 \mathrm{E}+0 \odot \quad 3.73127070 \mathrm{E}+0 \odot \quad 4.57669027 \mathrm{E}-02-9.06507146 \mathrm{E}-05$

7.73391065E-08-2.38150322E-11 8.47358554E+04 1.60375566E+@1

$\begin{array}{lllllll}\text { FE4CO FE 4C } 10 \quad 1 \quad \text { G } & 300.000 & 5000.000 & 995.043\end{array}$

$1.18334392 \mathrm{E}+01 \quad 5.93810062 \mathrm{E}-03-2.97229736 \mathrm{E}-06 \quad 5.36726022 \mathrm{E}-10-3.41227976 \mathrm{E}-14$

$1.05975241 \mathrm{E}+05-1.58740794 \mathrm{E}+01 \quad 3.63926344 \mathrm{E}+00 \quad 6.39661566 \mathrm{E}-02-1.28267591 \mathrm{E}-04$

1.09821721E-07-3.38577070E-11 1.06363955E+05 1.73764368E+01

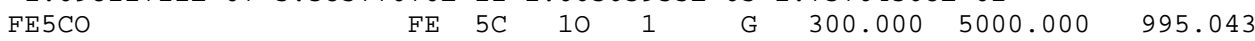

$1.42571686 \mathrm{E}+01 \quad 7.02247704 \mathrm{E}-03-3.59005571 \mathrm{E}-06 \quad 6.56145684 \mathrm{E}-10-4.20461850 \mathrm{E}-14$

$1.27714275 \mathrm{E}+05-2.57985671 \mathrm{E}+01 \quad 3.55420611 \mathrm{E}+00 \quad 8.29837993 \mathrm{E}-02-1.67750098 \mathrm{E}-04$

$1.43906571 \mathrm{E}-07-4.43907929 \mathrm{E}-11$ 1.28213733E+05 1.75906222E+01

$\begin{array}{lllllllll}\text { FE6C0 } & \text { FE } & 6 C & 10 & 1 & \text { G } & 300.000 & 5000.000 & 995.043\end{array}$

$1.62945667 \mathrm{E}+01 \quad 8.39915868 \mathrm{E}-03-4.28117399 \mathrm{E}-06 \quad 7.84011858 \mathrm{E}-10-5.03426296 \mathrm{E}-14$

$1.47865823 \mathrm{E}+05-3.01766182 \mathrm{E}+01 \quad 3.48249765 \mathrm{E}+0 \odot \quad 1.01924271 \mathrm{E}-01-2.08614048 \mathrm{E}-04$

$1.80125834 \mathrm{E}-07-5.57723394 \mathrm{E}-11 \quad 1.48335238 \mathrm{E}+05 \quad 2.11172358 \mathrm{E}+01$

$\begin{array}{lllllllll}\text { FE7C0 } & \text { FE } & 7 C & 10 & 1 & \text { G } & 300.000 & 5000.000 & 995.043\end{array}$

$1.87136527 \mathrm{E}+01 \quad 9.54235359 \mathrm{E}-03-4.93887186 \mathrm{E}-06 \quad 9.11950240 \mathrm{E}-10-5.88634780 \mathrm{E}-14$

$1.69852166 \mathrm{E}+05-4.12713737 \mathrm{E}+01 \quad 3.40812130 \mathrm{E}+00 \quad 1.21365854 \mathrm{E}-01-2.49330180 \mathrm{E}-04$

$2.15449945 \mathrm{E}-07-6.67235246 \mathrm{E}-11$ 1.70408150E+05 1.99811463E+01

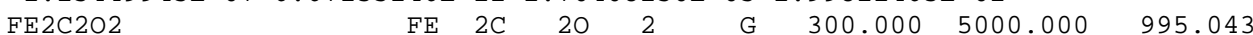

$1.08772172 \mathrm{E}+01 \quad 6.28436064 \mathrm{E}-03-2.87066985 \mathrm{E}-06 \quad 4.94249306 \mathrm{E}-10-3.04499524 \mathrm{E}-14$

$2.87072604 \mathrm{E}+04-1.65875888 \mathrm{E}+01 \quad 3.57208533 \mathrm{E}+\odot \odot \quad 4.86221947 \mathrm{E}-02-8.62483406 \mathrm{E}-\odot 5$

$6.94575859 \mathrm{E}-08-2.06488195 \mathrm{E}-11 \quad 2.95188720 \mathrm{E}+04 \quad 1.53922897 \mathrm{E}+01$

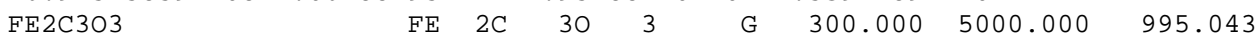

1.41893398E+01 9.05445523E-03-4.16203325E-06 7.25981504E-10-4.52141745E-14

$6.33070884 \mathrm{E}+03-2.85455772 \mathrm{E}+01 \quad 3.43758984 \mathrm{E}+00 \quad 7.56102976 \mathrm{E}-02-1.39669415 \mathrm{E}-\Theta 4$

1.15081960E-07-3.46979065E-11 7.31520860E+03 1.74672422E+01

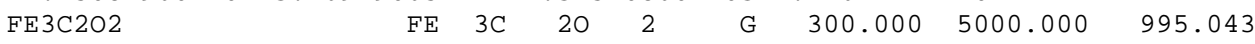

1.35015273E+01 6.62138319E-03-2.99483727E-06 5.57525626E-10-3.66883513E-14

$6.17089243 \mathrm{E}+\odot 4-2.19220703 \mathrm{E}+01 \quad 3.22725975 \mathrm{E}+\odot \odot \quad 7.57196672 \mathrm{E}-02-1.49061053 \mathrm{E}-\odot 4$ 


\section{Part IV: The Mechanism for Iron Nanoparticle Formation (0.3 atm).}

\author{
1. FEC404+CO<=>FEC505 \\ 2. $\mathrm{FEC} 303+\mathrm{CO}<=>\mathrm{FEC} 404$ \\ 3. $\mathrm{FEC} 303+\mathrm{FE}<=>\mathrm{FE} 2 \mathrm{C} 2 \mathrm{O} 2+\mathrm{CO}$ \\ 4. $\mathrm{FEC} 303+\mathrm{FE} 2<=>\mathrm{FE} 3 \mathrm{C} 2 \mathrm{O} 2+\mathrm{CO}$ \\ 5. $\mathrm{FEC} 303+\mathrm{FECO}<=>\mathrm{FE} 2 \mathrm{C} 303+\mathrm{CO}$ \\ 6. $\mathrm{FEC} 2 \mathrm{O} 2+\mathrm{CO}<=>\mathrm{FEC} 303$ \\ 7. $\mathrm{FEC} 2 \mathrm{O} 2+\mathrm{FE}<=>\mathrm{FE} 2 \mathrm{CO}+\mathrm{CO}$ \\ 8. $\mathrm{FEC} 2 \mathrm{O} 2+\mathrm{FE} 2<=>\mathrm{FE} 3 \mathrm{CO}+\mathrm{CO}$ \\ 9. $\mathrm{FEC} 2 \mathrm{O} 2+\mathrm{FE} 3<=>\mathrm{FE} 4 \mathrm{CO}+\mathrm{CO}$ \\ 10. $\mathrm{FEC} 2 \mathrm{O} 2+\mathrm{FE} 4<=>\mathrm{FE} 5 \mathrm{CO}+\mathrm{CO}$ \\ 11. FEC202+FE5 $<=>$ FE 6 CO + CO \\ 12. $\mathrm{FEC} 2 \mathrm{O} 2+\mathrm{FE} 6<=>\mathrm{FE} 7 \mathrm{CO}+\mathrm{CO}$ \\ 13. FEC202+FECO $<=>\mathrm{FE} 2 \mathrm{C} 2 \mathrm{O} 2+\mathrm{CO}$ \\ 14. $\mathrm{FEC} 2 \mathrm{O} 2+\mathrm{FE} 2 \mathrm{CO}<=>\mathrm{FE} 3 \mathrm{C} 2 \mathrm{O} 2+\mathrm{CO}$ \\ 15. FEC2O2+FEC2O2<=>FE2C $303+C 0$ \\ 16. FE2C $202+\mathrm{CO}<=>\mathrm{FE} 2 \mathrm{C} 303$ \\ 17. $\mathrm{FE} 2 \mathrm{C} 2 \mathrm{O} 2+\mathrm{FE}<=>\mathrm{FE} 3 \mathrm{CO}+\mathrm{CO}$ \\ 18. FE2C2O2+FE2 $<=>F E 4 C O+C O$ \\ 19. $\mathrm{FE} 2 \mathrm{C} 2 \mathrm{O} 2+\mathrm{FE} 3<=>\mathrm{FE} 5 \mathrm{CO}+\mathrm{CO}$ \\ 20. $\mathrm{FE} 2 \mathrm{C} 2 \mathrm{O} 2+\mathrm{FE} 4<=>\mathrm{FE} 6 \mathrm{CO}+\mathrm{CO}$ \\ 21. $\mathrm{FE} 2 \mathrm{C} 2 \mathrm{O} 2+\mathrm{FE} 5<=>\mathrm{FE} 7 \mathrm{CO}+\mathrm{CO}$ \\ 22. FE2C $202+\mathrm{FECO}<=>\mathrm{FE} 3 \mathrm{C} 2 \mathrm{O} 2+\mathrm{CO}$ \\ 23. $\mathrm{FE} 3 \mathrm{C} 2 \mathrm{O} 2+\mathrm{FE}<=>\mathrm{FE} 4 \mathrm{CO}+\mathrm{CO}$ \\ 24. $\mathrm{FE} 2 \mathrm{C} 303+\mathrm{FE}<=>\mathrm{FE} 3 \mathrm{C} 2 \mathrm{O} 2+\mathrm{CO}$ \\ 25. $\mathrm{FECO}+\mathrm{CO}<=>\mathrm{FEC} 2 \mathrm{O} 2$ \\ 26. $\mathrm{FECO}+\mathrm{FE}<=>\mathrm{FE} 2 \mathrm{CO}$ \\ 27. $\mathrm{FECO}+\mathrm{FECO}<=>\mathrm{FE} 2 \mathrm{CO}+\mathrm{CO}$ \\ 28. $\mathrm{FE}+\mathrm{CO}<=>\mathrm{FECO}$ \\ 29. $\mathrm{FE}+\mathrm{FECO}<=>\mathrm{FE} 2+\mathrm{CO}$ \\ 30. FE $2+\mathrm{FECO}<=>\mathrm{FE} 3+\mathrm{CO}$ \\ 31. $\mathrm{FE} 3+\mathrm{FECO}<=>\mathrm{FE} 4+\mathrm{CO}$ \\ 32. FE $4+\mathrm{FECO}<=>\mathrm{FE} 5+\mathrm{CO}$ \\ 33. FE5+FECO $<=>\mathrm{FE} 6+\mathrm{CO}$ \\ 34. $\mathrm{FE} 6+\mathrm{FECO}<=>\mathrm{FE} 7+\mathrm{CO}$ \\ 35. $\mathrm{FE} 7+\mathrm{FECO}<=>\mathrm{BO} 1+\mathrm{CO}$ \\ 36. $\mathrm{FE} 2+\mathrm{CO}<=>\mathrm{FE} 2 \mathrm{CO}$ \\ 37. $\mathrm{FE} 2 \mathrm{CO}+\mathrm{CO}<=>\mathrm{FE} 2 \mathrm{C} 2 \mathrm{O} 2$ \\ 38. $\mathrm{FE} 2 \mathrm{CO}+\mathrm{FE}<=>\mathrm{FE} 3+\mathrm{CO}$ \\ 39. FE2 $+\mathrm{FE} 2 \mathrm{CO}<=>\mathrm{FE} 4+\mathrm{CO}$ \\ 40. FE2CO+FE3 $<=>F E 5+C O$ \\ 41. FE2CO+FE4 $<=>$ FE $6+C 0$ \\ 42. FE2 $\mathrm{CO}+\mathrm{FE} 5<=>\mathrm{FE} 7+\mathrm{CO}$ \\ 43. $\mathrm{FE} 2 \mathrm{CO}+\mathrm{FE} 6<=>\mathrm{B} 01+\mathrm{CO}$ \\ 44. FE2CO+FE $7=>0 \cdot 875 \mathrm{~B} 01+\odot \cdot 125 \mathrm{~B} 02+\mathrm{CO}$ \\ 45. FE2CO+FECO $<=>\mathrm{FE} 3 \mathrm{CO}+\mathrm{CO}$ \\ 46. $\mathrm{FE} 2 \mathrm{CO}+\mathrm{FE} 2 \mathrm{CO}<=>\mathrm{FE} 4 \mathrm{CO}+\mathrm{CO}$ \\ 47. $\mathrm{FE} 3+\mathrm{CO}<=>\mathrm{FE} 3 \mathrm{CO}$ \\ 48. $\mathrm{FE} 3 \mathrm{CO}+\mathrm{CO}<=>\mathrm{FE} 3 \mathrm{C} 2 \mathrm{O} 2$ \\ 49. $\mathrm{FE} 3 \mathrm{CO}+\mathrm{FE}<=>\mathrm{FE} 4+\mathrm{CO}$ \\ 50. FE3CO+FE2 $<=>$ FE5 + CO \\ 51. FE3CO+FE3 $<=>F E 6+C O$ \\ 52. $\mathrm{FE} 3 \mathrm{CO}+\mathrm{FE} 4<=>\mathrm{FE} 7+\mathrm{CO}$ \\ 53. FE3CO+FE5 $<=>B O 1+C O$ \\ 54. $\mathrm{FE} 3 \mathrm{CO}+\mathrm{FECO}<=>\mathrm{FE} 4 \mathrm{CO}+\mathrm{CO}$ \\ 55. FE4+CO $<=>$ FE 4 CO \\ 56. $\mathrm{FE} 4 \mathrm{CO}+\mathrm{FE}<=>\mathrm{FE} 5+\mathrm{CO}$ \\ 57. FE4CO+FE2 $<=>\mathrm{FE} 6+\mathrm{CO}$ \\ 58. $\mathrm{FE} 4 \mathrm{CO}+\mathrm{FE} 3<=>\mathrm{FE} 7+\mathrm{CO}$ \\ 59. $\mathrm{FE} 4 \mathrm{CO}+\mathrm{FE} 4<=>\mathrm{B} 01+\mathrm{CO}$ \\ 60. $\mathrm{FE} 5+\mathrm{CO}<=>\mathrm{FE} 5 \mathrm{CO}$ \\ 61. $\mathrm{FE} 5 \mathrm{CO}+\mathrm{FE}<=>\mathrm{FE} 6+\mathrm{CO}$ \\ 62. FE $5 \mathrm{CO}+\mathrm{FE} 2<=>\mathrm{FE} 7+\mathrm{CO}$ \\ 63. FE5 $00+F E 3<=>B 01+\mathrm{CO}$ \\ 64. $\mathrm{FE} 6+\mathrm{CO}<=>\mathrm{FE} 6 \mathrm{CO}$
}

\begin{tabular}{|c|c|c|}
\hline $1.52 \mathrm{E}+49$ & -12.3 & 9486.0 \\
\hline $4.67 E+35$ & -7.6 & 4419.0 \\
\hline $5.39 E+12$ & 0.5 & 0.0 \\
\hline $5.57 \mathrm{E}+12$ & 0.5 & 0.0 \\
\hline $7.87 \mathrm{E}+12$ & 0.5 & 0.0 \\
\hline $8.43 E+38$ & -8.9 & 4564.0 \\
\hline $5.76 \mathrm{E}+12$ & 0.5 & 4.0 \\
\hline $5.14 \mathrm{E}+12$ & 0.5 & 0.0 \\
\hline $4.93 E+12$ & 0.5 & 0.0 \\
\hline $4.97 \mathrm{E}+12$ & 0.5 & 0.0 \\
\hline $5.11 \mathrm{E}+12$ & 0.5 & $\odot . \odot$ \\
\hline $5.25 \mathrm{E}+12$ & 0.5 & 0.0 \\
\hline $8.23 E+12$ & 0.5 & 0.0 \\
\hline $6.09 \mathrm{E}+12$ & 0.5 & 0.0 \\
\hline $6.42 \mathrm{E}+12$ & 0.5 & 0.0 \\
\hline $5.98 \mathrm{E}+25$ & -5.3 & 0.0 \\
\hline $2.82 \mathrm{E}+12$ & 0.5 & 0.0 \\
\hline $2.84 \mathrm{E}+12$ & 0.5 & 0.0 \\
\hline $2.41 E+12$ & 0.5 & 0.0 \\
\hline $2.05 \mathrm{E}+12$ & 0.5 & 0.0 \\
\hline $2.27 \mathrm{E}+12$ & 0.5 & 0.0 \\
\hline $4.67 \mathrm{E}+12$ & 0.5 & 0.0 \\
\hline $7.78 \mathrm{E}+12$ & 0.5 & 0.0 \\
\hline $5.60 \mathrm{E}+12$ & 0.5 & 0.0 \\
\hline $3.79 E+33$ & -7.1 & 3324.0 \\
\hline $5.95 \mathrm{E}+30$ & -5.8 & 2975.0 \\
\hline $6.84 \mathrm{E}+12$ & 0.5 & 0.0 \\
\hline 1. $64 \mathrm{E}+14$ & -2.1 & 630.0 \\
\hline $5.55 \mathrm{E}+12$ & 0.5 & 7.0 \\
\hline $2.68 \mathrm{E}+12$ & 0.5 & 0.0 \\
\hline $2.57 \mathrm{E}+12$ & 0.5 & 0.0 \\
\hline $2.58 \mathrm{E}+12$ & 0.5 & 0.0 \\
\hline $2.69 \mathrm{E}+12$ & 0.5 & 0.0 \\
\hline $2.78 \mathrm{E}+12$ & 0.5 & 0.0 \\
\hline 2. $90 \mathrm{E}+12$ & 0.5 & 0.0 \\
\hline $5.30 \mathrm{E}+25$ & -4.9 & 2127.0 \\
\hline $5.0 \odot E+32$ & -7.4 & 2543.0 \\
\hline $3.07 \mathrm{E}+12$ & 0.5 & 7.0 \\
\hline $2.94 \mathrm{E}+12$ & 0.5 & 0.0 \\
\hline 1. $01 \mathrm{E}+13$ & 0.5 & 0.0 \\
\hline 1. $19 \mathrm{E}+13$ & 0.5 & 0.0 \\
\hline 1. $24 \mathrm{E}+13$ & 0.5 & 0.0 \\
\hline 1. $33 \mathrm{E}+13$ & 0.5 & 0.0 \\
\hline 1. $42 \mathrm{E}+13$ & 0.5 & 0.0 \\
\hline $4.19 \mathrm{E}+12$ & 0.5 & 0.0 \\
\hline $5.06 \mathrm{E}+12$ & 0.5 & 0.0 \\
\hline $7.95 \mathrm{E}+28$ & -6.3 & 2553.0 \\
\hline $6.41 E+24$ & -4.8 & 202.0 \\
\hline $2.23 E+12$ & 0.5 & 0.0 \\
\hline 1. $79 \mathrm{E}+12$ & 0.5 & 0.0 \\
\hline 1. $45 \mathrm{E}+12$ & 0.5 & 0.0 \\
\hline 1. $22 \mathrm{E}+12$ & 0.5 & 0.0 \\
\hline 1. $21 \mathrm{E}+12$ & 0.5 & 0.0 \\
\hline $3.52 \mathrm{E}+12$ & 0.5 & $\odot .0$ \\
\hline $1.50 \mathrm{E}+36$ & -8.4 & 3212.0 \\
\hline 1. $89 \mathrm{E}+12$ & 0.5 & 0.0 \\
\hline 1. $65 \mathrm{E}+12$ & 0.5 & 0.0 \\
\hline $1.18 \mathrm{E}+12$ & 0.5 & 0.0 \\
\hline 1. $14 \mathrm{E}+12$ & 0.5 & $\odot .0$ \\
\hline $3.87 E+39$ & -9.4 & 3608.0 \\
\hline 1. $97 \mathrm{E}+12$ & 0.5 & 0.0 \\
\hline 1. $48 \mathrm{E}+12$ & 0.5 & 0.0 \\
\hline 1. $.44 \mathrm{E}+12$ & 0.5 & $\odot . \odot$ \\
\hline $1.45 \mathrm{E}+33$ & -7.2 & $2024 . \odot$ \\
\hline
\end{tabular}


65. $\mathrm{FE} 6 \mathrm{CO}+\mathrm{FE}<=>\mathrm{FE} 7+\mathrm{CO}$

66. $\mathrm{FE} 6 \mathrm{CO}+\mathrm{FE} 2<=>\mathrm{B} 01+\mathrm{CO}$

67. $\mathrm{FE} 7+\mathrm{CO}<=>\mathrm{FE} 7 \mathrm{CO}$

68. $\mathrm{FE} 7 \mathrm{CO}+\mathrm{FE}<=>\mathrm{B} 01+\mathrm{C} 0$

69. $\mathrm{FE}+\mathrm{FE}+\mathrm{M}<=>\mathrm{FE} 2+\mathrm{M}$

70. $F E+F E 2<=>F E 3$

71. FE+FE3 $<=>F E 4$

72. $F E+F E 4<=>F E 5$

73. $\mathrm{FE}+\mathrm{FE} 5<=>\mathrm{FE} 6$

74. $\mathrm{FE}+\mathrm{FE} 6<=>\mathrm{FE} 7$

75. $F E+F E 7<=>B 01$

76. $\mathrm{FE} 2+\mathrm{FE} 2<=>\mathrm{FE} 4$

77. FE2+FE2 $<=>F E+F E 3$

78. $\mathrm{FE} 2+\mathrm{FE} 3<=>\mathrm{FE} 5$

79. FE2+FE3<=>FE+FE4

80. FE2+FE4<=>FE6

81. FE2+FE4<=>FE3+FE3

82. FE2+FE4<=>FE+FE5

83. FE2+FE5 $<=>$ FE 7

84. FE2+FE5 $<=>$ FE+FE 6

85. $\mathrm{FE} 2+\mathrm{FE} 5<=>\mathrm{FE} 3+\mathrm{FE} 4$

86. FE2 $+F E 6<=>B 01$

87. $\mathrm{FE} 2+\mathrm{FE} 7=>0.875 \mathrm{~B} 01+\odot .125 \mathrm{~B} 02$

88. FE3+FE3 $<=>F E 6$

89. FE3+FE3 $<=>F E+F E 5$

90. FE3+FE4 $<=>F E 7$

91. FE3+FE4<=>FE+FE6

92. $\mathrm{FE} 3+\mathrm{FE} 5<=>\mathrm{B} 01$

93. $\mathrm{FE} 3+\mathrm{FE} 6=>0.875 \mathrm{~B} 01+\odot .125 \mathrm{~B} 02$

94. $F E 3+F E 7=>0.750 B 01+0.250 B 02$

95. FE4+FE4 $<=>B 01$

96. $\mathrm{FE} 4+\mathrm{FE} 5=>0.875 \mathrm{~B} 01+0.125 \mathrm{~B} 02$

97. $\mathrm{FE} 4+\mathrm{FE} 6=>0.750 \mathrm{~B} 01+0.250 \mathrm{~B} 02$

98. $\mathrm{FE} 4+\mathrm{FE} 7=>0.625 \mathrm{~B} 01+0.375 \mathrm{~B} 02$

99. $\mathrm{FE} 5+\mathrm{FE} 5=>0.750 \mathrm{~B} 01+0.250 \mathrm{~B} 02$

100. $\mathrm{FE} 5+\mathrm{FE} 6=>0.625 \mathrm{~B} 01+0.375 \mathrm{~B} 02$

101. $\mathrm{FE} 5+\mathrm{FE} 7=>0.500 \mathrm{~B} 01+0.500 \mathrm{~B} 02$

102. $\mathrm{FE} 6+\mathrm{FE} 6=>0.500 \mathrm{~B} 01+\odot .500 \mathrm{~B} 02$

103. $\mathrm{FE} 6+\mathrm{FE} 7=>0.375 \mathrm{~B} 01+0.625 \mathrm{BO} 2$

104. $\mathrm{FE} 7+\mathrm{FE} 7=>0.250 \mathrm{~B} 01+0.750 \mathrm{~B} \odot 2$

105. $\mathrm{FE}+\mathrm{B} 01$ $=>.8749999999999999 \mathrm{~B} 01+.1250000000000001 \mathrm{~B} 02$

106. $\mathrm{FE}+\mathrm{B} \odot 2$ $=>.93750000000000000 \mathrm{~B} 02+.06250000000000000 \mathrm{~B} 03$

107. $\mathrm{FE}+\mathrm{B} 03$ $=>.9687500000000000 \mathrm{~B} 03+.03125000000000000 \mathrm{~B} 04$

108. $\mathrm{FE}+\mathrm{B} 04$ $=>.9843750000000001 \mathrm{~B} 04+.0156249999999999 \mathrm{~B} 05$

109. FE+B05 $=>.9921875000000000 \mathrm{~B} 05+.0078125000000000 \mathrm{~B} 06$

110. $\mathrm{FE}+\mathrm{B} 06$ $=>.9960937500000000 \mathrm{~B} 06+.00390625000000000 \mathrm{~B} \odot 7$

111. $\mathrm{FE}+\mathrm{B} 07$ $=>.9980468750000000 \mathrm{~B} 07+.00195312500000000 \mathrm{~B} 08$

112. $\mathrm{FE}+\mathrm{B} 08$ $=>$. $9990234375000000 \mathrm{~B} 08+.0009765625000000 \mathrm{~B} \odot 9$

113. $\mathrm{FE}+\mathrm{B} 09$ $=>$. $999511718750000 \odot \mathrm{B} 09+.000488281250000 \odot \mathrm{B} 10$

114. $\mathrm{FE}+\mathrm{B} 10$ $=>.9997558593750000 \mathrm{~B} 10+.0002441406250000 \mathrm{~B} 11$

115. $\mathrm{FE}+\mathrm{B} 11$ $=>.9998779296875001 \mathrm{~B} 11+.0001220703124999 \mathrm{~B} 12$

116. $\mathrm{FE}+\mathrm{B} 12$ $=>.9999389648437500 \mathrm{~B} 12+.0000610351562500 \mathrm{~B} 13$

117. FE+B13 $=>$. $9999694824218750 \mathrm{~B} 13+.0000305175781250 \mathrm{~B} 14$

118. $\mathrm{FE}+\mathrm{B} 14$ $=>.9999847412109374 \mathrm{~B} 14+.0000152587890626 \mathrm{~B} 15$

119. FE+B15 $=>.9999923706054688 B 15+.0000076293945313 B 16$

120. $\mathrm{FE}+\mathrm{B} 16$

\begin{tabular}{|c|c|c|}
\hline 1. $94 \mathrm{E}+12$ & 0.5 & 0.0 \\
\hline 1. $48 \mathrm{E}+12$ & 0.5 & 0.0 \\
\hline $5.10 \mathrm{E}+21$ & -4.0 & 0.0 \\
\hline 1. $92 \mathrm{E}+12$ & 0.5 & 0.0 \\
\hline $7.89 \mathrm{E}+17$ & -0.52 & 14.8 \\
\hline $4.60 \mathrm{E}+23$ & -4.1 & 1717.0 \\
\hline $1.94 \mathrm{E}+30$ & -6.2 & 2898.0 \\
\hline $1.58 \mathrm{E}+38$ & -8.6 & 4846.0 \\
\hline $2.81 \mathrm{E}+40$ & -8.8 & 5965.0 \\
\hline $1.49 \mathrm{E}+34$ & -6.6 & 5008.0 \\
\hline 1. $62 \mathrm{E}+37$ & -7.6 & 5725.0 \\
\hline 1. $09 \mathrm{E}+28$ & -5.0 & 2814.0 \\
\hline $3.41 \mathrm{E}+12$ & 0.5 & 2.0 \\
\hline $4.71 E+33$ & -7.1 & 3759.0 \\
\hline $6.91 \mathrm{E}+12$ & 0.4 & 97.0 \\
\hline $4.72 \mathrm{E}+37$ & -8.1 & 5132.0 \\
\hline $4.65 \mathrm{E}+20$ & -1.8 & 7751.0 \\
\hline 1. $00 \mathrm{E}+13$ & 0.3 & 145.0 \\
\hline $2.43 E+27$ & -4.7 & 3733.0 \\
\hline $3.45 \mathrm{E}+12$ & 0.5 & 59.0 \\
\hline $7.79 \mathrm{E}+25$ & -3.1 & 15404.0 \\
\hline $9.63 \mathrm{E}+23$ & -3.3 & 2804.0 \\
\hline $1.38 \mathrm{E}+13$ & 0.5 & 0.0 \\
\hline $5.27 \mathrm{E}+38$ & -8.8 & 4749.0 \\
\hline $6.63 \mathrm{E}+15$ & -0.7 & 780.0 \\
\hline $8.84 \mathrm{E}+37$ & -8.1 & 5540.0 \\
\hline $8.98 \mathrm{E}+14$ & -0.4 & 653.0 \\
\hline $7.07 \mathrm{E}+30$ & -5.6 & 4340.0 \\
\hline 1. $28 \mathrm{E}+13$ & 0.5 & 0.0 \\
\hline $1.32 \mathrm{E}+13$ & 0.5 & $\odot . \odot$ \\
\hline $3.40 \mathrm{E}+34$ & -6.9 & 5123.0 \\
\hline 1. $24 \mathrm{E}+13$ & 0.5 & 0.0 \\
\hline 1. $27 \mathrm{E}+13$ & 0.5 & 0.0 \\
\hline 1. $31 \mathrm{E}+13$ & 0.5 & 0.0 \\
\hline $1.26 \mathrm{E}+13$ & 0.5 & 0.0 \\
\hline 1. $28 \mathrm{E}+13$ & 0.5 & 0.0 \\
\hline $1.31 \mathrm{E}+13$ & 0.5 & 0.0 \\
\hline 1. $30 \mathrm{E}+13$ & 0.5 & $\odot .0$ \\
\hline 1. $32 \mathrm{E}+13$ & 0.5 & 0.0 \\
\hline $1.33 \mathrm{E}+13$ & 0.5 & 0.0 \\
\hline 1. $63 \mathrm{E}+14$ & 0.5 & 0.0 \\
\hline $2.17 \mathrm{E}+14$ & 0.5 & 0.0 \\
\hline $3.01 \mathrm{E}+14$ & 0.5 & 0.0 \\
\hline $4.29 \mathrm{E}+14$ & 0.5 & 0.0 \\
\hline $6.24 \mathrm{E}+14$ & 0.5 & 0.0 \\
\hline $9.22 \mathrm{E}+14$ & 0.5 & 0.0 \\
\hline $1.38 \mathrm{E}+15$ & 0.5 & $\odot .0$ \\
\hline 2. $09 \mathrm{E}+15$ & 0.5 & 0.0 \\
\hline $3.20 \mathrm{E}+15$ & 0.5 & 0.0 \\
\hline $4.92 \mathrm{E}+15$ & 0.5 & 0.0 \\
\hline $7.62 \mathrm{E}+15$ & 0.5 & $\odot . \odot$ \\
\hline 1. $19 \mathrm{E}+16$ & 0.5 & 0.0 \\
\hline $1.85 \mathrm{E}+16$ & 0.5 & 0.0 \\
\hline $2.91 \mathrm{E}+16$ & 0.5 & 0.0 \\
\hline $4.57 \mathrm{E}+16$ & 0.5 & 0.0 \\
\hline $7.19 \mathrm{E}+16$ & 0.5 & $\odot . \odot$ \\
\hline
\end{tabular}




\begin{tabular}{|c|c|c|c|c|}
\hline 121. & $\begin{array}{l}\mathrm{FE}+\mathrm{B} 17 \\
=>.9999980926513672 \mathrm{~B} 17+.0000019073486328 \mathrm{~B} 18\end{array}$ & $1.13 \mathrm{E}+17$ & 0.5 & 0.0 \\
\hline 122. & $\begin{array}{l}\text { FE+B18 } \\
=>.9999990463256836 B 18+.0000009536743164 B 19\end{array}$ & $1.79 \mathrm{E}+17$ & 0.5 & 0.0 \\
\hline 123. & $\begin{array}{l}\text { FE+B19 } \\
=>.9999995231628418 B 19+.0000004768371582 B 20\end{array}$ & $2.83 \mathrm{E}+17$ & 0.5 & 0.0 \\
\hline 124. & $\begin{array}{l}\text { FE2+B01 } \\
=>.7499999999999999 \mathrm{~B} 01+.2500000000000001 \mathrm{~B} 02\end{array}$ & $1.43 \mathrm{E}+14$ & 0.5 & $0 . \odot$ \\
\hline 125. & $\begin{array}{l}\text { FE2+BO2 } \\
=>.8749999999999999 B 02+.1250000000000001 \mathrm{~B} 03\end{array}$ & $1.83 E+14$ & 0.5 & $\odot . \odot$ \\
\hline 126. & $\begin{array}{l}\text { FE2+B०3 } \\
=>.93750000000000000 \mathrm{~B} \odot 3+.0625000000000 \odot \odot \odot \mathrm{B} \odot 4\end{array}$ & $2.44 \mathrm{E}+14$ & 0.5 & 0.0 \\
\hline 127. & 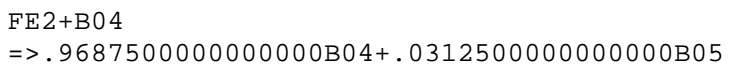 & $3.38 \mathrm{E}+14$ & 0.5 & $\odot . \odot$ \\
\hline 128. & $\begin{array}{l}\text { FE2+B०5 } \\
=>.9843750000000001 B 05+.0156249999999999 B \odot 66\end{array}$ & $4.82 \mathrm{E}+14$ & 0.5 & 0.0 \\
\hline 129. & 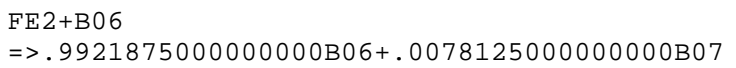 & $7.00 \mathrm{E}+14$ & 0.5 & 0.0 \\
\hline 130. & $\begin{array}{l}\text { FE2+B०7 } \\
=>.9960937500000 \odot \odot \odot B \odot 7+.00390625000 \odot \odot \odot \odot \odot B \odot 8\end{array}$ & $1.03 \mathrm{E}+15$ & 0.5 & $\odot . \odot$ \\
\hline 131. & $\begin{array}{l}\text { FE2+B०8 } \\
=>.9980468750000 \odot \odot \odot B \odot 8+.001953125000 \odot \odot \odot \odot B \odot 9\end{array}$ & $1.55 \mathrm{E}+15$ & 0.5 & $\odot . \odot$ \\
\hline 132. & $\begin{array}{l}\text { FE2+B०9 } \\
=>.9990234375000000 \mathrm{~B} \odot 9+.0009765625000000 \mathrm{~B} 10\end{array}$ & $2.35 \mathrm{E}+15$ & 0.5 & $0 . \odot$ \\
\hline 133. & $\begin{array}{l}\text { FE2+B10 } \\
=>.9995117187500000 \mathrm{~B} 10+.0004882812500000 \mathrm{~B} 11\end{array}$ & $3.59 \mathrm{E}+15$ & 0.5 & 0.0 \\
\hline 134. & $\begin{array}{l}\text { FE2+B11 } \\
=>.9997558593750000 B 11+.0002441406250000 B 12\end{array}$ & $5.52 \mathrm{E}+15$ & 0.5 & 0.0 \\
\hline 135. & $\begin{array}{l}\text { FE2+B12 } \\
=>.9998779296875001 B 12+.0001220703124999 B 13\end{array}$ & $8.56 \mathrm{E}+15$ & 0.5 & 0.0 \\
\hline 136. & $\begin{array}{l}\text { FE2+B13 } \\
=>.9999389648437500 \mathrm{~B} 13+.0000610351562500 \mathrm{~B} 14\end{array}$ & $1.33 \mathrm{E}+16$ & 0.5 & 0.0 \\
\hline 137. & $\begin{array}{l}\text { FE2+B14 } \\
=>.9999694824218750 B 14+.0000305175781250 B 15\end{array}$ & $2.08 \mathrm{E}+16$ & 0.5 & 0.0 \\
\hline 138. & $\begin{array}{l}\text { FE2+B15 } \\
=>.9999847412109374 \mathrm{~B} 15+.0000152587890626 \mathrm{~B} 16\end{array}$ & $3.26 \mathrm{E}+16$ & 0.5 & 0.0 \\
\hline 139. & $\begin{array}{l}\text { FE2+B16 } \\
=>.9999923706054688 B 16+.0000076293945313 B 17\end{array}$ & $5.13 \mathrm{E}+16$ & 0.5 & 0.0 \\
\hline 140. & $\begin{array}{l}\text { FE2+B17 } \\
=>.9999961853027344 \mathrm{~B} 17+.0000038146972656 \mathrm{~B} 18\end{array}$ & 8. $08 \mathrm{E}+16$ & 0.5 & 0.0 \\
\hline 141. & $\begin{array}{l}\text { FE2+B18 } \\
=>.9999980926513672 B 18+.0000019073486328 B 19\end{array}$ & $1.27 \mathrm{E}+17$ & 0.5 & 0.0 \\
\hline 142. & $\begin{array}{l}\text { FE2+B19 } \\
=>.9999990463256836 B 19+.0000009536743164 B 20\end{array}$ & $2.01 \mathrm{E}+17$ & 0.5 & 0.0 \\
\hline 143. & $\begin{array}{l}\text { FE3+B०1 } \\
=>.6250000000000001 B 01+.3749999999999999 \mathrm{~B} \odot 22\end{array}$ & $1.37 \mathrm{E}+14$ & 0.5 & 0.0 \\
\hline 144. & $\begin{array}{l}\text { FE3+BO2 } \\
=>.8124999999999999 B 02+.1875000000000001 B 03\end{array}$ & $1.68 \mathrm{E}+14$ & 0.5 & $\odot . \odot$ \\
\hline 145. & 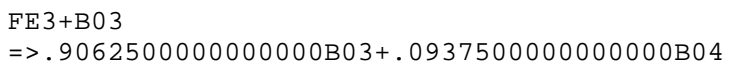 & $2.19 \mathrm{E}+14$ & 0.5 & 0.0 \\
\hline 146. & 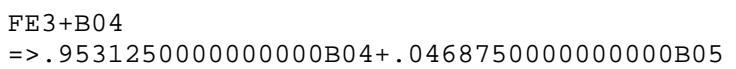 & $2.98 \mathrm{E}+14$ & 0.5 & 0.0 \\
\hline 147. & $\begin{array}{l}\text { FE3+B05 } \\
=>.97656250000000000 \mathrm{~B} 05+.02343750000000000 \mathrm{~B} 06\end{array}$ & $4.18 \mathrm{E}+14$ & 0.5 & 0.0 \\
\hline 148. & $\begin{array}{l}\text { FE3+B०6 } \\
=>.9882812500000000 \mathrm{~B} \odot 6+.0117187500000000 \mathrm{~B} \odot 7\end{array}$ & $6.00 \mathrm{E}+14$ & 0.5 & 0.0 \\
\hline 149. & $\begin{array}{l}\text { FE3+BO7 } \\
=>.9941406250000000 \mathrm{~B} 07+.0058593750000000 \mathrm{~B} \odot 8\end{array}$ & $8.79 E+14$ & 0.5 & $\odot . \odot$ \\
\hline 150. & $\begin{array}{l}\text { FE3+B०8 } \\
=>.9970703124999999 B \odot 8+.0029296875000001 B \odot 9\end{array}$ & $1.31 \mathrm{E}+15$ & 0.5 & 0.0 \\
\hline 151. & $\begin{array}{l}\text { FE3+B०9 } \\
=>.998535156250000 \odot B \odot 9+.00146484375000 \odot \odot B 10\end{array}$ & $1.97 \mathrm{E}+15$ & 0.5 & 0.0 \\
\hline 152. & $\begin{array}{l}\text { FE3+B10 } \\
=>.9992675781250000 \mathrm{~B} 10+.0007324218750000 \mathrm{~B} 11\end{array}$ & $2.99 \mathrm{E}+15$ & 0.5 & 0.0 \\
\hline 153. & $\begin{array}{l}\text { FE3+B11 } \\
=>.9996337890625001 B 11+.0003662109374999 B 12\end{array}$ & $4.59 \mathrm{E}+15$ & 0.5 & $\odot . \odot$ \\
\hline 154. & $\begin{array}{l}\text { FE3+B12 } \\
=>.9998168945312500 \mathrm{~B} 12+.0001831054687500 \mathrm{~B} 13\end{array}$ & $7.08 \mathrm{E}+15$ & 0.5 & $\odot . \odot$ \\
\hline 155. & $\begin{array}{l}\text { FE3+B13 } \\
=>.9999084472656250 B 13+.0000915527343750 B 14\end{array}$ & $1.10 \mathrm{E}+16$ & 0.5 & 0.0 \\
\hline
\end{tabular}


156. FE3+B14

$=>.9999542236328125 B 14+.0000457763671875 B 15$

157. FE3+B15

$=>.9999771118164063 B 15+.0000228881835938 B 16$

158. FE3+B16

$=>.9999885559082031 B 16+.0000114440917969 B 17$

159. FE3+B17

$=>.9999942779541016 \mathrm{~B} 17+.0000057220458984 \mathrm{~B} 18$

160. FE3+B18

=> . 9999971389770509B18+. .0000028610229491B19

161. FE3+B19

$=>.9999985694885254 \mathrm{~B} 19+.0000014305114746 \mathrm{~B} 20$

162. FE4+BO1

$=>.5000000000000000 \mathrm{~B} 01+.50000000000000000 \mathrm{~B} 02$

163. FE4+BO2

$=>.7499999999999999 B \odot 2+.2500000000000001 \mathrm{~B} 03$

164. FE4+BO3

$=>.8749999999999999 \mathrm{~B} 03+.1250000000000001 \mathrm{~B} 04$

165. FE4+B०4

$=>.9375000000000000 \mathrm{~B} 04+.06250000000000000 \mathrm{~B} 05$

166. FE4+B05

$=>.96875000000000000 \mathrm{~B} 05+.03125000000000000 \mathrm{~B} 06$

167. FE4+BO6

$=>.9843750000000001 \mathrm{~B} 06+.0156249999999999 \mathrm{~B} 07$

168. FE4+BO7

$=>.99218750000000000 \mathrm{~B} 07+.00781250000000000 \mathrm{~B} 08$

169. FE4+B08

$=>.99609375000000000 \mathrm{~B} 08+.00390625000000000 \mathrm{~B} 09$

170. FE4+BO9

$=>.9980468750000000 \mathrm{~B} 09+.0019531250000000 \mathrm{~B} 10$

171. FE4+B10

$=>.9990234375000000 \mathrm{~B} 10+.0009765625000000 \mathrm{~B} 11$

172. FE4+B11

$=>.9995117187500000 \mathrm{~B} 11+.0004882812500000 \mathrm{~B} 12$

173. FE4+B12

$=>.9997558593750000 \mathrm{~B} 12+.0002441406250000 \mathrm{~B} 13$

174. FE4+B13

$=>.9998779296875001 B 13+.0001220703124999 B 14$

175. FE4+B14

$=>.9999389648437500 \mathrm{~B} 14+.0000610351562500 \mathrm{~B} 15$

176. FE4+B15

=> . $9999694824218750 \mathrm{~B} 15+.0000305175781250 \mathrm{~B} 16$

177. FE4+B16

=> . 9999847412109374B16+. . $0000152587890626 \mathrm{~B} 17$

178. FE4+B17

$=>.9999923706054688 \mathrm{~B} 17+.0000076293945313 \mathrm{~B} 18$

179. FE4+B18

$=>$. 9999961853027344B18+. .0000038146972656B19

180. FE4+B19

$=>.9999980926513672 \mathrm{~B} 19+.0000019073486328 \mathrm{~B} 20$

181. FE5+B01

$=>.3749999999999999 \mathrm{~B} 01+.6250000000000000 \mathrm{~B} 02$

182. $\mathrm{FE} 5+\mathrm{B} \odot 2$

$=>.6875000000000001 \mathrm{~B} 02+.3124999999999999 \mathrm{~B} 03$

183. FE5+BO3

$=>.8437500000000000 \mathrm{~B} 03+.15625000000000000 \mathrm{~B} 04$

184. FE5+BO4

$=>.9218750000000000 \mathrm{~B} 04+.0781250000000000 \mathrm{~B} 05$

185. FE5+B05

$=>.960937500000000000 \mathrm{~B} 05+.03906250000000000 \mathrm{~B} 06$

186. FE5+B06

$=>.9804687500000000 \mathrm{~B} 06+.01953125000000000 \mathrm{~B} 07$

187. FE5+BO7

$=>.9902343750000000 \mathrm{~B} 07+.0097656250000000 \mathrm{~B} 08$

188. FE5+B08

=> . 9951171875000000B08+. . 0048828125000000B०9

189. FE5+B09

$=>.9975585937500000 \mathrm{~B} 09+.00244140625000000 \mathrm{~B} 10$

190. FE5+B10

$=>.9987792968750000 \mathrm{~B} 10+.0012207031250000 \mathrm{~B} 11$

191. FE5+B11

\begin{tabular}{|c|c|c|}
\hline 1. $71 \mathrm{E}+16$ & 0.5 & $\odot . \odot$ \\
\hline $2.68 \mathrm{E}+16$ & 0.5 & 0.0 \\
\hline $4.21 E+16$ & 0.5 & $\odot . \odot$ \\
\hline $6.62 \mathrm{E}+16$ & 0.5 & $\odot . \odot$ \\
\hline 1. $04 \mathrm{E}+17$ & 0.5 & $\odot . \odot$ \\
\hline 1. $65 \mathrm{E}+17$ & 0.5 & $\odot . \odot$ \\
\hline 1. $34 \mathrm{E}+14$ & 0.5 & $\odot . \odot$ \\
\hline $1.61 \mathrm{E}+14$ & 0.5 & $\odot .0$ \\
\hline $2.05 E+14$ & 0.5 & $\odot . \odot$ \\
\hline $2.74 \mathrm{E}+14$ & 0.5 & 0.0 \\
\hline $3.80 \mathrm{E}+14$ & 0.5 & $\odot . \odot$ \\
\hline $5.41 \mathrm{E}+14$ & 0.5 & 0.0 \\
\hline $7.86 \mathrm{E}+14$ & 0.5 & $\odot . \odot$ \\
\hline 1. $16 \mathrm{E}+15$ & 0.5 & $\odot . \odot$ \\
\hline 1. $74 \mathrm{E}+15$ & 0.5 & 0.0 \\
\hline $2.64 \mathrm{E}+15$ & 0.5 & 0.0 \\
\hline 4. $.3 E+15$ & 0.5 & $\odot . \odot$ \\
\hline $6.20 \mathrm{E}+15$ & 0.5 & $\odot . \odot$ \\
\hline $9.61 \mathrm{E}+15$ & 0.5 & 0.0 \\
\hline $1.50 \mathrm{E}+16$ & 0.5 & $\odot . \odot$ \\
\hline $2.34 \mathrm{E}+16$ & 0.5 & $\odot . \odot$ \\
\hline $3.66 \mathrm{E}+16$ & 0.5 & $\odot . \odot$ \\
\hline $5.76 \mathrm{E}+16$ & 0.5 & 0.0 \\
\hline $9.06 \mathrm{E}+16$ & 0.5 & $\odot . \odot$ \\
\hline $1.43 E+17$ & 0.5 & $\odot . \odot$ \\
\hline $6.68 \mathrm{E}+13$ & 0.5 & $\odot . \odot$ \\
\hline $7.80 E+13$ & 0.5 & $\odot . \odot$ \\
\hline $9.77 \mathrm{E}+13$ & 0.5 & $\odot . \odot$ \\
\hline 1. $29 \mathrm{E}+14$ & 0.5 & $0 . \odot$ \\
\hline 1. $77 \mathrm{E}+14$ & 0.5 & $\odot . \odot$ \\
\hline $2.50 \mathrm{E}+14$ & 0.5 & 0.0 \\
\hline $3.61 \mathrm{E}+14$ & 0.5 & $\odot . \odot$ \\
\hline $5.30 \mathrm{E}+14$ & 0.5 & $\odot . \odot$ \\
\hline $7.91 \mathrm{E}+14$ & 0.5 & 0.0 \\
\hline 1. $19 \mathrm{E}+15$ & 0.5 & $\odot . \odot$ \\
\hline 1. $82 \mathrm{E}+15$ & 0.5 & 0.0 \\
\hline
\end{tabular}




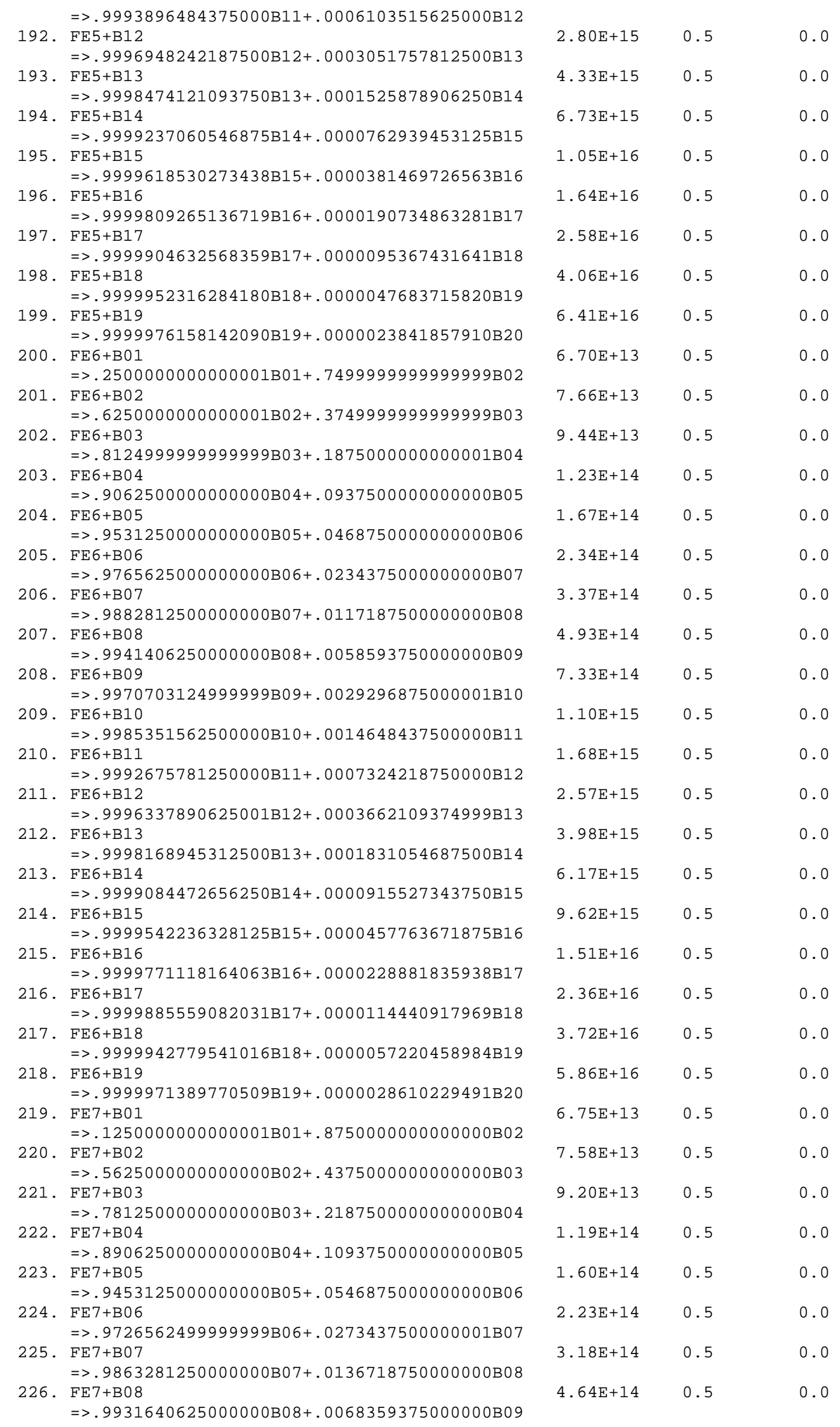


227. FE7+BO9
$=>.9965820312500001 B 09+.0034179687499999 B 10$

228. FE7+B10

$=>.9982910156250000 \mathrm{~B} 10+.0017089843750000 \mathrm{~B} 11$

229. $\mathrm{FE} 7+\mathrm{B} 11$

$=>.9991455078125000 \mathrm{~B} 11+.0008544921875000 \mathrm{~B} 12$

230. $\mathrm{FE} 7+\mathrm{B} 12$

$=>.9995727539062500 \mathrm{~B} 12+.0004272460937500 \mathrm{~B} 13$

231. FE7+B13

$=>.9997863769531250 \mathrm{~B} 13+.0002136230468750 \mathrm{~B} 14$

232. FE7+B14

$=>.9998931884765625 B 14+.0001068115234375 B 15$

233. FE7+B15

$=>.9999465942382813 B 15+.0000534057617188 B 16$

234. FE7+B16

$=>.9999732971191407 \mathrm{~B} 16+.0000267028808593 \mathrm{~B} 17$

235. FE7+B17

$=>.9999866485595703 B 17+.0000133514404297 B 18$

236. FE7+B18

$=>.9999933242797852 \mathrm{~B} 18+.0000066757202148 \mathrm{~B} 19$

237. FE7+B19

$=>.9999966621398925 B 19+.0000033378601075 B 20$

238. $\mathrm{B} 01+\mathrm{B} 01=>\mathrm{B} 02$

239. $\mathrm{B} 01+\mathrm{B} 02$

$=>.5000000000000000 \mathrm{~B} 02+.5000000000000000 \mathrm{~B} 03$

240. B01+B03

$=>.7499999999999999 \mathrm{~B} 03+.2500000000000001 \mathrm{~B} 04$

241. B01+B०4

$=>.8749999999999999 \mathrm{~B} 04+.1250000000000001 \mathrm{~B} 05$

242. B $01+\mathrm{B} 05$

$=>.9375000000000000 \mathrm{~B} 05+.06250000000000000 \mathrm{~B} 06$

243. $\mathrm{B} 01+\mathrm{B} 06$

$=>.9687500000000000 \mathrm{~B} 06+.0312500000000000 \mathrm{~B} 07$

244. B०1+BO7

$=>.9843750000000001 \mathrm{~B} 07+.0156249999999999 \mathrm{~B} 08$

245. B01+B08

$=>.9921875000000000 \mathrm{~B} 08+.0078125000000000 \mathrm{~B} 09$

246. B01+B09

$=>.9960937500000000 \mathrm{~B} 09+.0039062500000000 \mathrm{~B} 10$

247. B01+B10

$=>.99804687500000000 \mathrm{~B} 10+.0019531250000000 \mathrm{~B} 11$

248. B01+B11

$=>.9990234375000000 \mathrm{~B} 11+.0009765625000000 \mathrm{~B} 12$

249. B01+B12

$=>.9995117187500000 \mathrm{~B} 12+.0004882812500000 \mathrm{~B} 13$

250. B01+B13

$=>.9997558593750000 \mathrm{~B} 13+.0002441406250000 \mathrm{~B} 14$

251. B01+B14

$=>.9998779296875001 \mathrm{~B} 14+.0001220703124999 \mathrm{~B} 15$

252. B01+B15

$=>.9999389648437500 \mathrm{~B} 15+.0000610351562500 \mathrm{~B} 16$

253. B01+B16

$=>.9999694824218750 \mathrm{~B} 16+.0000305175781250 \mathrm{~B} 17$

254. B01+B17

$=>.9999847412109374 \mathrm{~B} 17+.0000152587890626 \mathrm{~B} 18$

255. B01+B18

$=>.9999923706054688 B 18+.0000076293945313 B 19$

256. B01+B19

$=>.9999961853027344 \mathrm{~B} 19+.0000038146972656 \mathrm{~B} 20$

257. $\mathrm{B} \odot 2+\mathrm{B} \odot 2=>\mathrm{B} \odot 3$

258. $\mathrm{B} 02+\mathrm{B} 03$

$=>.500000000000000000 \mathrm{~B} 03+.500000000000000000 \mathrm{~B} 04$

259. $\mathrm{B} 02+\mathrm{B} 04$

$=>.7499999999999999 \mathrm{~B} 04+.2500000000000001 \mathrm{~B} 05$

260. B०2+B०5

$=>.8749999999999999 \mathrm{~B} 05+.1250000000000001 \mathrm{~B} 06$

261. B०2+B06

$=>.9375000000000000 \mathrm{~B} 06+.062500000000000000 \mathrm{~B} 07$

262. B०2+BO7

$=>.9687500000000000 \mathrm{~B} 07+.03125000000000000 \mathrm{~B} 08$

263. $\mathrm{B} 02+\mathrm{B} 08$

\begin{tabular}{|c|c|c|}
\hline $6.88 \mathrm{E}+14$ & 0.5 & 0.0 \\
\hline 1. $03 \mathrm{E}+15$ & 0.5 & $\odot . \odot$ \\
\hline $1.57 \mathrm{E}+15$ & 0.5 & $\odot . \odot$ \\
\hline $2.40 \mathrm{E}+15$ & 0.5 & $\odot . \odot$ \\
\hline 3. $70 \mathrm{E}+15$ & 0.5 & $\odot . \odot$ \\
\hline $5.74 \mathrm{E}+15$ & 0.5 & $\odot . \odot$ \\
\hline $8.94 \mathrm{E}+15$ & 0.5 & $\odot . \odot$ \\
\hline 1. $40 \mathrm{E}+16$ & 0.5 & $\odot . \odot$ \\
\hline $2.19 \mathrm{E}+16$ & 0.5 & $\odot . \odot$ \\
\hline $3.45 \mathrm{E}+16$ & 0.5 & $\odot . \odot$ \\
\hline $5.43 \mathrm{E}+16$ & 0.5 & $\odot . \odot$ \\
\hline 2. $\odot 4 \mathrm{E}+12$ & 0.5 & $\odot . \odot$ \\
\hline $2.26 \mathrm{E}+12$ & 0.5 & 0.0 \\
\hline 2. $70 \mathrm{E}+12$ & 0.5 & $\odot . \odot$ \\
\hline $3.45 \mathrm{E}+12$ & 0.5 & $\odot . \odot$ \\
\hline $4.61 E+12$ & 0.5 & $\odot . \odot$ \\
\hline $6.39 \mathrm{E}+12$ & 0.5 & $\odot . \odot$ \\
\hline $9 \cdot 10 \mathrm{E}+12$ & 0.5 & $\odot . \odot$ \\
\hline 1. $32 \mathrm{E}+13$ & 0.5 & $\odot . \odot$ \\
\hline 1. $95 \mathrm{E}+13$ & 0.5 & $\odot . \odot$ \\
\hline $2.93 \mathrm{E}+13$ & 0.5 & $\odot . \odot$ \\
\hline 4. $44 \mathrm{E}+13$ & 0.5 & 0.0 \\
\hline $6.78 \mathrm{E}+13$ & 0.5 & $\odot . \odot$ \\
\hline 1. $04 \mathrm{E}+14$ & 0.5 & 0.0 \\
\hline 1. $62 \mathrm{E}+14$ & 0.5 & $\odot . \odot$ \\
\hline $2.52 \mathrm{E}+14$ & 0.5 & $\odot .0$ \\
\hline $3.93 \mathrm{E}+14$ & 0.5 & 0.0 \\
\hline $6.17 \mathrm{E}+14$ & 0.5 & $\odot . \odot$ \\
\hline $9.69 \mathrm{E}+14$ & 0.5 & 0.0 \\
\hline $1.53 \mathrm{E}+15$ & 0.5 & $\odot . \odot$ \\
\hline $2.29 \mathrm{E}+12$ & 0.5 & $\odot . \odot$ \\
\hline $2.54 \mathrm{E}+12$ & 0.5 & $\odot . \odot$ \\
\hline 3. $03 \mathrm{E}+12$ & 0.5 & $\odot .0$ \\
\hline 3. $87 \mathrm{E}+12$ & 0.5 & $\odot . \odot$ \\
\hline $5 \cdot 18 \mathrm{E}+12$ & 0.5 & $\odot . \odot$ \\
\hline $7.18 \mathrm{E}+12$ & 0.5 & $\odot . \odot$ \\
\hline 1. $02 \mathrm{E}+13$ & 0.5 & $\odot . \odot$ \\
\hline
\end{tabular}




\begin{tabular}{|c|c|c|c|c|}
\hline 264 . & $\begin{array}{l}\mathrm{B} 02+\mathrm{B} 09 \\
=>.9921875000000000 \mathrm{~B} 09+.0078125000000000 \odot \mathrm{B} 10\end{array}$ & $1.48 \mathrm{E}+13$ & 0.5 & 0.0 \\
\hline 265. & $\begin{array}{l}\text { B } 22+B 10 \\
=>.9960937500000000 \mathrm{~B} 10+.0039062500000000 \mathrm{~B} 11\end{array}$ & $2.19 \mathrm{E}+13$ & 0.5 & 0.0 \\
\hline 266. & $\begin{array}{l}\text { B } 02+B 11 \\
=>.998046875000000 \odot B 11+.0019531250000 \odot \odot \odot B 12\end{array}$ & $3.29 \mathrm{E}+13$ & 0.5 & 0.0 \\
\hline 267. & $\begin{array}{l}\text { B } 22+B 12 \\
=>.9990234375000000 \mathrm{~B} 12+.0009765625000000 \mathrm{~B} 13\end{array}$ & $4.98 \mathrm{E}+13$ & 0.5 & 0.0 \\
\hline 268. & $\begin{array}{l}\mathrm{B} 02+\mathrm{B} 13 \\
=>.9995117187500000 \mathrm{~B} 13+.0004882812500000 \mathrm{~B} 14\end{array}$ & $7.61 E+13$ & 0.5 & $\odot . \odot$ \\
\hline 269. & $\begin{array}{l}\text { B } 22+B 14 \\
=>.9997558593750000 \mathrm{~B} 14+.0002441406250000 \mathrm{~B} 15\end{array}$ & $1.17 \mathrm{E}+14$ & 0.5 & 0.0 \\
\hline 270. & $\begin{array}{l}\text { B02+B15 } \\
=>.9998779296875001 B 15+.0001220703124999 B 16\end{array}$ & $1.82 \mathrm{E}+14$ & 0.5 & 0.0 \\
\hline 271. & $\begin{array}{l}\text { B02+B16 } \\
=>.9999389648437500 \mathrm{~B} 16+.0000610351562500 \mathrm{~B} 17\end{array}$ & $2.83 E+14$ & 0.5 & 0.0 \\
\hline 272 . & $\begin{array}{l}\mathrm{B} 02+\mathrm{B} 17 \\
=>.9999694824218750 \mathrm{~B} 17+.0000305175781250 \mathrm{~B} 18\end{array}$ & $4.42 \mathrm{E}+14$ & 0.5 & 0.0 \\
\hline 273. & $\begin{array}{l}\text { B02+B18 } \\
=>.9999847412109374 \mathrm{~B} 18+.0000152587890626 \mathrm{~B} 19\end{array}$ & $6.92 \mathrm{E}+14$ & 0.5 & $\odot . \odot$ \\
\hline 274. & $\begin{array}{l}\text { B०2+B19 } \\
=>.9999923706054688 B 19+.0000076293945313 \text { B } 20\end{array}$ & $1.09 \mathrm{E}+15$ & 0.5 & $\odot . \odot$ \\
\hline 275 . & $\mathrm{B} 03+\mathrm{B} \odot 3=>\mathrm{B} \odot 4$ & $2.57 \mathrm{E}+12$ & 0.5 & 0.0 \\
\hline 276. & $\begin{array}{l}\text { B०3+B०4 } \\
=>.5000000000000000 \mathrm{~B} \odot 4+.5000000000000000 \mathrm{~B} \odot 5\end{array}$ & $2.85 \mathrm{E}+12$ & 0.5 & $\odot . \odot$ \\
\hline 277. & $\begin{array}{l}\text { B०3+B०5 } \\
=>.7499999999999999 B 05+.2500000000000001 B 06\end{array}$ & $3 \cdot 41 \mathrm{E}+12$ & 0.5 & 0.0 \\
\hline 278. & $\begin{array}{l}\text { B०3+B०6 } \\
=>.8749999999999999 B 06+.1250000000000001 B 07\end{array}$ & $4.34 \mathrm{E}+12$ & 0.5 & 0.0 \\
\hline 279. & $\begin{array}{l}\text { B०3+B०7 } \\
=>.9375000000000000 \mathrm{~B} 07+.0625000000000000 \mathrm{~B} 08\end{array}$ & $5.81 \mathrm{E}+12$ & 0.5 & $0 . \odot$ \\
\hline 280. & $\begin{array}{l}\text { B०3+B०8 } \\
=>.9687500000000000 \mathrm{~B} 08+.0312500000000000 \mathrm{~B} \odot 9\end{array}$ & $8.05 E+12$ & 0.5 & 0.0 \\
\hline 281. & $\begin{array}{l}\text { B०3+B०9 } \\
=>.9843750000000001 B 09+.0156249999999999 B 10\end{array}$ & $1.15 \mathrm{E}+13$ & 0.5 & 0.0 \\
\hline 282. & $\begin{array}{l}\text { B०3+B10 } \\
=>.9921875000000 \odot \odot \odot B 10+.0078125000000 \odot \odot \odot B 11\end{array}$ & $1.67 \mathrm{E}+13$ & 0.5 & 0.0 \\
\hline 283. & $\begin{array}{l}\text { B०3+B11 } \\
=>.9960937500000000 \mathrm{~B} 11+.0039062500000000 \mathrm{~B} 12\end{array}$ & $2.46 \mathrm{E}+13$ & 0.5 & 0.0 \\
\hline 284. & $\begin{array}{l}\text { B } 03+\mathrm{B} 12 \\
=>.9980468750000000 \mathrm{~B} 12+.0019531250000 \odot \odot \odot \mathrm{B} 13\end{array}$ & $3.69 \mathrm{E}+13$ & 0.5 & 0.0 \\
\hline 285. & $\begin{array}{l}\text { B०3+B13 } \\
=>.9990234375000000 \mathrm{~B} 13+.000976562500000 \odot \mathrm{B} 14\end{array}$ & $5.59 E+13$ & 0.5 & 0.0 \\
\hline 286. & $\begin{array}{l}\text { B०3+B14 } \\
=>.9995117187500000 \mathrm{~B} 14+.0004882812500000 \mathrm{~B} 15\end{array}$ & $8.54 \mathrm{E}+13$ & 0.5 & 0.0 \\
\hline 287. & $\begin{array}{l}\text { B०3+B15 } \\
=>.999755859375000 \odot B 15+.000244140625000 \odot B 16\end{array}$ & $1.32 \mathrm{E}+14$ & 0.5 & 0.0 \\
\hline 288. & $\begin{array}{l}\text { B03+B16 } \\
=>.9998779296875001 B 16+.0001220703124999 B 17\end{array}$ & $2.04 \mathrm{E}+14$ & 0.5 & 0.0 \\
\hline 289. & $\begin{array}{l}\text { B०3+B17 } \\
=>.9999389648437500 \mathrm{~B} 17+.0000610351562500 \mathrm{~B} 18\end{array}$ & $3 \cdot 17 \mathrm{E}+14$ & 0.5 & 0.0 \\
\hline 290. & $\begin{array}{l}\text { B03+B18 } \\
=>.9999694824218750 \mathrm{~B} 18+.0000305175781250 \mathrm{~B} 19\end{array}$ & 4. $96 \mathrm{E}+14$ & 0.5 & $\odot . \odot$ \\
\hline 291. & $\begin{array}{l}\text { B03+B19 } \\
=>.9999847412109374 B 19+.0000152587890626 B 20\end{array}$ & $7.77 \mathrm{E}+14$ & 0.5 & 0.0 \\
\hline 292. & $\mathrm{~B} 04+\mathrm{B} 04=>\mathrm{B} 05$ & $2.89 \mathrm{E}+12$ & 0.5 & 0.0 \\
\hline 293. & $\begin{array}{l}\text { B०4+B०5 } \\
=>.5000000000000000 \mathrm{~B} 05+.5000000000000000 \mathrm{~B} 06\end{array}$ & $3.20 \mathrm{E}+12$ & 0.5 & 0.0 \\
\hline 294. & $\begin{array}{l}\text { B०4+B०6 } \\
=>.7499999999999999 B 06+.2500000000000001 B 07\end{array}$ & $3.82 \mathrm{E}+12$ & 0.5 & 0.0 \\
\hline 295. & $\begin{array}{l}\text { B०4+B०7 } \\
=>.8749999999999999 \mathrm{~B} 07+.1250000000000001 \mathrm{~B} 08\end{array}$ & $4.88 \mathrm{E}+12$ & 0.5 & 0.0 \\
\hline 296. & $\begin{array}{l}\text { B०4+B०8 } \\
=>.9375000000000000 B 08+.0625000000000000 \mathrm{~B} 09\end{array}$ & $6.52 \mathrm{E}+12$ & 0.5 & 0.0 \\
\hline 297. & $\begin{array}{l}\text { B०4+B०9 } \\
=>.9687500000000000 \mathrm{~B} 09+.0312500000000000 \mathrm{~B} 10\end{array}$ & $9.04 \mathrm{E}+12$ & 0.5 & 0.0 \\
\hline 298. & $\begin{array}{l}\text { B०4+B10 } \\
=>.9843750000000001 \mathrm{~B} 10+.0156249999999999 \mathrm{~B} 11\end{array}$ & $1.29 \mathrm{E}+13$ & 0.5 & $\odot . \odot$ \\
\hline 299. & $\begin{array}{l}\text { B०4+B11 } \\
=>.9921875000000000 \mathrm{~B} 11+.0078125000000000 \mathrm{~B} 12\end{array}$ & $1.87 \mathrm{E}+13$ & 0.5 & 0.0 \\
\hline
\end{tabular}


300. $\mathrm{B} 04+\mathrm{B} 12$

$=>.9960937500000000 \mathrm{~B} 12+.0039062500000000 \mathrm{~B} 13$

301. B04+B13

$=>.9980468750000000 \mathrm{~B} 13+.0019531250000000 \mathrm{~B} 14$

302. $\mathrm{B} 04+\mathrm{B} 14$

$=>.9990234375000000 \mathrm{~B} 14+.0009765625000000 \mathrm{~B} 15$

303. B04+B15

$=>.9995117187500000 \mathrm{~B} 15+.0004882812500000 \mathrm{~B} 16$

304. B०4+B16

$=>.9997558593750000 \mathrm{~B} 16+.0002441406250000 \mathrm{~B} 17$

305. B04+B17

$=>.9998779296875001 B 17+.0001220703124999 B 18$

306. B04+B18

$=>.9999389648437500 \mathrm{~B} 18+.0000610351562500 \mathrm{~B} 19$

307. B04+B19

$=>.9999694824218750 \mathrm{~B} 19+.0000305175781250 \mathrm{~B} 20$

308. $\mathrm{B} 05+\mathrm{B} 05=>\mathrm{B} 06$

309. $\mathrm{B} 05+\mathrm{B} 06$

$=>.5000000000000000 \mathrm{~B} 06+.50000000000000000 \mathrm{~B} 07$

310. B05+B07

$=>.7499999999999999 B 07+.2500000000000001 B 08$

311. B०5+B०8

$=>.8749999999999999 \mathrm{~B} 08+.1250000000000001 \mathrm{~B} 09$

312. В05+B09

$=>.9375000000000000 \mathrm{~B} 09+.06250000000000000 \mathrm{~B} 10$

313. B05+B10

$=>.9687500000000000 \mathrm{~B} 10+.031250000000000000 \mathrm{~B} 11$

314. B05+B11

$=>.9843750000000001 B 11+.0156249999999999 B 12$

315. B05+B12

$=>.9921875000000000 \mathrm{~B} 12+.00781250000000000 \mathrm{~B} 13$

316. B05+B13

$=>.9960937500000000 \mathrm{~B} 13+.0039062500000000 \mathrm{~B} 14$

317. B05+B14

$=>.9980468750000000 \mathrm{~B} 14+.0019531250000000 \mathrm{~B} 15$

318. B05+B15

$=>.9990234375000000 \mathrm{~B} 15+.0009765625000000 \mathrm{~B} 16$

319. B०5+B16

$=>.9995117187500000 \mathrm{~B} 16+.0004882812500000 \mathrm{~B} 17$

320. B05+B17

$=>.9997558593750000 \mathrm{~B} 17+.0002441406250000 \mathrm{~B} 18$

321. B05+B18

$=>.9998779296875001 \mathrm{~B} 18+.0001220703124999 \mathrm{~B} 19$

322. B05+B19

$=>.9999389648437500 \mathrm{~B} 19+.0000610351562500 \mathrm{~B} 20$

323. $\mathrm{B} 06+\mathrm{B} 06=>\mathrm{B} 07$

324. $\mathrm{B} 06+\mathrm{B} 07$

$=>.5000000000000000 \mathrm{~B} 07+.50000000000000000 \mathrm{~B} 08$

325. B०6+B08

$=>.7499999999999999 \mathrm{~B} 08+.2500000000000001 \mathrm{~B} 09$

326. B०6+B०9

$=>.8749999999999999 B 09+.1250000000000001 B 10$

327. B06+B10

$=>.9375000000000000 \mathrm{~B} 10+.06250000000000000 \mathrm{~B} 11$

328. B06+B11

$=>.9687500000000000 \mathrm{~B} 11+.0312500000000000 \mathrm{~B} 12$

329. B06+B12

$=>.9843750000000001 \mathrm{~B} 12+.0156249999999999 \mathrm{~B} 13$

330. B06+B13

$=>.99218750000000000 \mathrm{~B} 13+.00781250000000000 \mathrm{~B} 14$

331. B06+B14

$=>.99609375000000000 \mathrm{~B} 14+.00390625000000000 \mathrm{~B} 15$

332. B06+B15

$=>.99804687500000000 \mathrm{~B} 15+.00195312500000000 \mathrm{~B} 16$

333. $\mathrm{B} 06+\mathrm{B} 16$

$=>.9990234375000000 \mathrm{~B} 16+.0009765625000000 \mathrm{~B} 17$

334. B06+B17

$=>.9995117187500000 \mathrm{~B} 17+.0004882812500000 \mathrm{~B} 18$

335. B06+B18

$=>.9997558593750000 \mathrm{~B} 18+.0002441406250000 \mathrm{~B} 19$

336. B06+B19

\begin{tabular}{|c|c|c|}
\hline $2.76 \mathrm{E}+13$ & 0.5 & 0.0 \\
\hline $4.14 \mathrm{E}+13$ & 0.5 & 0.0 \\
\hline $6.27 \mathrm{E}+13$ & 0.5 & 0.0 \\
\hline $9.59 \mathrm{E}+13$ & 0.5 & $\odot . \odot$ \\
\hline $1.48 \mathrm{E}+14$ & $\odot .5$ & 0.0 \\
\hline $2.29 \mathrm{E}+14$ & 0.5 & 0.0 \\
\hline $3.56 \mathrm{E}+14$ & 0.5 & 0.0 \\
\hline $5.56 \mathrm{E}+14$ & 0.5 & 0.0 \\
\hline $\begin{array}{l}3.24 \mathrm{E}+12 \\
3.59 \mathrm{E}+12\end{array}$ & $\begin{array}{l}0.5 \\
0.5\end{array}$ & $\begin{array}{l}0.0 \\
0.0\end{array}$ \\
\hline $4.29 \mathrm{E}+12$ & 0.5 & $\odot . \odot$ \\
\hline $5.47 \mathrm{E}+12$ & 0.5 & $0 . \odot$ \\
\hline 7. $32 \mathrm{E}+12$ & 0.5 & $0 . \odot$ \\
\hline 1. $01 \mathrm{E}+13$ & 0.5 & $\odot . \odot$ \\
\hline 1. $44 \mathrm{E}+13$ & 0.5 & 0.0 \\
\hline $2.10 \mathrm{E}+13$ & 0.5 & $0 . \odot$ \\
\hline $3.10 \mathrm{E}+13$ & $\odot .5$ & $0 . \odot$ \\
\hline $4.65 E+13$ & 0.5 & $\odot . \odot$ \\
\hline $7.04 \mathrm{E}+13$ & 0.5 & 0.0 \\
\hline 1. $08 \mathrm{E}+14$ & 0.5 & 0.0 \\
\hline $1.66 \mathrm{E}+14$ & 0.5 & 0.0 \\
\hline $2.57 \mathrm{E}+14$ & 0.5 & 0.0 \\
\hline 4. $.0 \mathrm{E}+14$ & $\odot .5$ & 0.0 \\
\hline $3.64 \mathrm{E}+12$ & $\odot .5$ & $0 . \odot$ \\
\hline 4. $03 \mathrm{E}+12$ & 0.5 & 0.0 \\
\hline $4.82 E+12$ & 0.5 & 0.0 \\
\hline $6.14 \mathrm{E}+12$ & 0.5 & 0.0 \\
\hline $8.22 \mathrm{E}+12$ & 0.5 & 0.0 \\
\hline $1.14 \mathrm{E}+13$ & 0.5 & $0 . \odot$ \\
\hline $1.62 \mathrm{E}+13$ & 0.5 & $0 . \odot$ \\
\hline $2.36 \mathrm{E}+13$ & $\odot .5$ & 0.0 \\
\hline $3.48 \mathrm{E}+13$ & 0.5 & $0 . \odot$ \\
\hline $5.22 \mathrm{E}+13$ & 0.5 & 0.0 \\
\hline $7.90 \mathrm{E}+13$ & 0.5 & 0.0 \\
\hline $1.21 \mathrm{E}+14$ & 0.5 & $0 . \odot$ \\
\hline 1. $86 \mathrm{E}+14$ & $\odot .5$ & $\odot . \odot$ \\
\hline $2.88 \mathrm{E}+14$ & 0.5 & 0.0 \\
\hline
\end{tabular}




\begin{tabular}{|c|c|c|c|c|}
\hline \multirow{3}{*}{$\begin{array}{l}337 . \\
338 .\end{array}$} & \multicolumn{4}{|l|}{$=>.9998779296875001 B 19+.0001220703124999 B 20$} \\
\hline & $\mathrm{B} 07+\mathrm{B} 07=>\mathrm{B} 08$ & 4. $.9 \mathrm{E}+12$ & 0.5 & $\odot . \odot$ \\
\hline & 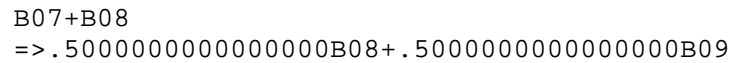 & $4.52 \mathrm{E}+12$ & 0.5 & 0.0 \\
\hline 339. & $\begin{array}{l}\text { B } 07+B 09 \\
=>.7499999999999999 B 09+.2500000000000001 B 10\end{array}$ & $5.41 \mathrm{E}+12$ & 0.5 & $0 . \odot$ \\
\hline 340. & $\begin{array}{l}\text { B०7+B10 } \\
=>.8749999999999999 B 10+.1250000000000001 B 11\end{array}$ & $6.90 \mathrm{E}+12$ & 0.5 & $\odot . \odot$ \\
\hline 341. & $\begin{array}{l}\text { B07+B11 } \\
=>.9375000000000000 \mathrm{~B} 11+.0625000000000000 \mathrm{~B} 12\end{array}$ & $9.23 \mathrm{E}+12$ & 0.5 & $\odot . \odot$ \\
\hline 342. & $\begin{array}{l}\text { B } 07+B 12 \\
=>.9687500000000000 B 12+.0312500000000000 B 13\end{array}$ & $1.28 \mathrm{E}+13$ & 0.5 & $\odot . \odot$ \\
\hline 343. & $\begin{array}{l}\text { B } 17+B 13 \\
=>.9843750000000001 B 13+.0156249999999999 B 14\end{array}$ & $1.82 \mathrm{E}+13$ & 0.5 & $\odot . \odot$ \\
\hline 344. & $\begin{array}{l}\text { B } 07+B 14 \\
=>.9921875000000000 \mathrm{~B} 14+.0078125000000000 \mathrm{~B} 15\end{array}$ & $2.65 \mathrm{E}+13$ & 0.5 & $\odot . \odot$ \\
\hline 345. & $\begin{array}{l}\text { B07+B15 } \\
=>.9960937500000000 B 15+.0039062500000000 B 16\end{array}$ & $3.91 \mathrm{E}+13$ & 0.5 & $\odot . \odot$ \\
\hline 346. & $\begin{array}{l}\text { B } 07+B 16 \\
=>.9980468750000000 B 16+.0019531250000 \odot \odot \odot B 17\end{array}$ & $5.86 \mathrm{E}+13$ & 0.5 & 0.0 \\
\hline 347. & $\begin{array}{l}\text { B०7+B17 } \\
=>.99902343750000 \odot \odot B 17+.000976562500000 \odot B 18\end{array}$ & $8.87 \mathrm{E}+13$ & 0.5 & $\odot . \odot$ \\
\hline 348. & $\begin{array}{l}\text { B } 07+B 18 \\
=>.9995117187500000 B 18+.0004882812500000 B 19\end{array}$ & $1.36 \mathrm{E}+14$ & 0.5 & $\odot . \odot$ \\
\hline 349. & $\begin{array}{l}\text { B०7+B19 } \\
=>.9997558593750000 B 19+.0002441406250000 B 20\end{array}$ & $2.09 \mathrm{E}+14$ & 0.5 & $\odot . \odot$ \\
\hline 350. & $\mathrm{~B} 08+\mathrm{B} 08=>\mathrm{B} 09$ & $4.59 E+12$ & 0.5 & $\odot . \odot$ \\
\hline 351. & $\begin{array}{l}\text { B०8+B०9 } \\
=>.5000000000000000 B 09+.5000000000000000 \mathrm{~B} 10\end{array}$ & $5.07 \mathrm{E}+12$ & 0.5 & $\odot . \odot$ \\
\hline 352. & $\begin{array}{l}\text { B08+B10 } \\
=>.7499999999999999 B 10+.2500000000000001 B 11\end{array}$ & $6.07 \mathrm{E}+12$ & 0.5 & $\odot . \odot$ \\
\hline 353. & $\begin{array}{l}\text { B08+B11 } \\
=>.8749999999999999 B 11+.1250000000000001 B 12\end{array}$ & $7.74 \mathrm{E}+12$ & 0.5 & $\odot . \odot$ \\
\hline 354. & 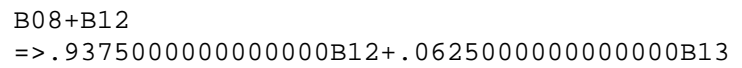 & 1. $04 \mathrm{E}+13$ & 0.5 & $\odot . \odot$ \\
\hline 355. & $\begin{array}{l}\text { B08+B13 } \\
=>.9687500000000000 \mathrm{~B} 13+.0312500000000000 \mathrm{~B} 14\end{array}$ & $1.44 \mathrm{E}+13$ & 0.5 & $\odot . \odot$ \\
\hline 356. & $\begin{array}{l}\text { B०8+B14 } \\
=>.9843750000000001 B 14+.0156249999999999 B 15\end{array}$ & $2.04 \mathrm{E}+13$ & 0.5 & 0.0 \\
\hline 357. & $\begin{array}{l}\text { B08+B15 } \\
=>.9921875000000000 B 15+.0078125000000000 B 16\end{array}$ & $2.97 \mathrm{E}+13$ & 0.5 & $\odot . \odot$ \\
\hline 358. & $\begin{array}{l}\text { B०8+B16 } \\
=>.99609375000000 \odot \odot B 16+.0039062500000 \odot \odot \odot B 17\end{array}$ & $4.39 E+13$ & 0.5 & $\odot . \odot$ \\
\hline 359. & $\begin{array}{l}\text { B08+B17 } \\
=>.9980468750000000 B 17+.0019531250000000 B 18\end{array}$ & $6.57 \mathrm{E}+13$ & 0.5 & $\odot . \odot$ \\
\hline 360. & $\begin{array}{l}\text { B०8+B18 } \\
=>.9990234375000000 B 18+.0009765625000000 B 19\end{array}$ & $9.96 \mathrm{E}+13$ & 0.5 & $\odot . \odot$ \\
\hline 361. & $\begin{array}{l}\text { B08+B19 } \\
=>.9995117187500000 B 19+.0004882812500000 B 20\end{array}$ & $1.52 \mathrm{E}+14$ & 0.5 & 0.0 \\
\hline 362. & $\mathrm{~B} 09+\mathrm{B} 09=>\mathrm{B} 10$ & $5.15 \mathrm{E}+12$ & 0.5 & 0.0 \\
\hline 363. & $\begin{array}{l}\text { B०9+B10 } \\
=>.5000000000000000 \mathrm{~B} 10+.5000000000000000 \mathrm{~B} 11\end{array}$ & $5.69 \mathrm{E}+12$ & 0.5 & $\odot . \odot$ \\
\hline 364. & $\begin{array}{l}\text { B09+B11 } \\
=>.7499999999999999 B 11+.2500000000000001 B 12\end{array}$ & $6.81 \mathrm{E}+12$ & 0.5 & $\odot . \odot$ \\
\hline 365. & $\begin{array}{l}\text { B०9+B12 } \\
=>.8749999999999999 B 12+.1250000000000001 B 13\end{array}$ & $8.69 E+12$ & 0.5 & 0.0 \\
\hline 366. & $\begin{array}{l}\text { B०9+B13 } \\
=>.9375000000000000 B 13+.0625000000000000 B 14\end{array}$ & $1.16 \mathrm{E}+13$ & 0.5 & $\odot . \odot$ \\
\hline 367. & $\begin{array}{l}\text { B०9+B14 } \\
=>.9687500000000000 \mathrm{~B} 14+.0312500000000000 \mathrm{~B} 15\end{array}$ & $1.61 \mathrm{E}+13$ & 0.5 & $\odot . \odot$ \\
\hline 368. & $\begin{array}{l}\text { B09+B15 } \\
=>.9843750000000001 B 15+.0156249999999999 B 16\end{array}$ & $2.29 \mathrm{E}+13$ & 0.5 & $\odot . \odot$ \\
\hline 369. & $\begin{array}{l}\text { B } 09+B 16 \\
=>.9921875000000000 B 16+.0078125000000000 B 17\end{array}$ & $3.33 E+13$ & 0.5 & $\odot . \odot$ \\
\hline 370. & $\begin{array}{l}\text { B09+B17 } \\
=>.9960937500000000 B 17+.0039062500000 \odot \odot \odot B 18\end{array}$ & $4.93 E+13$ & 0.5 & 0.0 \\
\hline 371. & $\begin{array}{l}\text { B०9+B18 } \\
=>.9980468750000000 \mathrm{~B} 18+.0019531250000000 \mathrm{~B} 19\end{array}$ & $7.38 \mathrm{E}+13$ & 0.5 & $\odot . \odot$ \\
\hline 372. & $\begin{array}{l}\text { B09+B19 } \\
=>.9990234375000000 B 19+.0009765625000000 B 20\end{array}$ & $1.12 \mathrm{E}+14$ & $\odot .5$ & $\odot . \odot$ \\
\hline 373. & $\mathrm{~B} 10+\mathrm{B} 10=>\mathrm{B} 11$ & $5.78 \mathrm{E}+12$ & 0.5 & 0.0 \\
\hline
\end{tabular}


374. $\mathrm{B} 10+\mathrm{B} 11$

$=>.5000000000000000 \mathrm{~B} 11+.5000000000000000 \mathrm{~B} 12$

375. $\mathrm{B} 10+\mathrm{B} 12$

$=>.7499999999999999 B 12+.2500000000000001 B 13$

376. $\mathrm{B} 10+\mathrm{B} 13$

$=>.8749999999999999 \mathrm{~B} 13+.12500000000000001 \mathrm{~B} 14$

377. B10+B14

$=>.9375000000000000 \mathrm{~B} 14+.06250000000000000 \mathrm{~B} 15$

378. $\mathrm{B} 10+\mathrm{B} 15$

$=>.9687500000000000 \mathrm{~B} 15+.03125000000000000 \mathrm{~B} 16$

379. B10+B16

$=>.9843750000000001 B 16+.0156249999999999 B 17$

380. B10+B17

$=>.9921875000000000 \mathrm{~B} 17+.0078125000000000 \mathrm{~B} 18$

381. B10+B18

$=>.9960937500000000 \mathrm{~B} 18+.0039062500000000 \mathrm{~B} 19$

382. $\mathrm{B} 10+\mathrm{B} 19$

$=>.9980468750000000 \mathrm{~B} 19+.00195312500000000 \mathrm{~B} 20$

383. $\mathrm{B} 11+\mathrm{B} 11=>\mathrm{B} 12$

384. $\mathrm{B} 11+\mathrm{B} 12$

$=>.50000000000000000 \mathrm{~B} 12+.50000000000000000 \mathrm{~B} 13$

385. B11+B13

$=>.7499999999999999 B 13+.2500000000000001 B 14$

386. B11+B14

$=>.8749999999999999 B 14+.1250000000000001 B 15$

387. B11+B15

$=>.9375000000000000 \mathrm{~B} 15+.062500000000000000 \mathrm{~B} 16$

388. B11+B16

$=>.9687500000000000 \mathrm{~B} 16+.0312500000000000 \mathrm{~B} 17$

389. $\mathrm{B} 11+\mathrm{B} 17$

$=>.9843750000000001 \mathrm{~B} 17+.0156249999999999 \mathrm{~B} 18$

390. B11+B18

$=>.99218750000000000 \mathrm{~B} 18+.00781250000000000 \mathrm{~B} 19$

391. B11+B19

$=>.99609375000000000 \mathrm{~B} 19+.00390625000000000 \mathrm{~B} 20$

392. $\mathrm{B} 12+\mathrm{B} 12=>\mathrm{B} 13$

393. $\mathrm{B} 12+\mathrm{B} 13$

$=>.5000000000000000 \mathrm{~B} 13+.5000000000000000 \mathrm{~B} 14$

394. B12+B14

$=>.7499999999999999 B 14+.2500000000000001 B 15$

395. B12+B15

$=>.8749999999999999 B 15+.1250000000000001 B 16$

396. B12+B16

$=>.9375000000000000 \mathrm{~B} 16+.0625000000000000 \mathrm{~B} 17$

397. B12+B17

$=>.9687500000000000 \mathrm{~B} 17+.03125000000000000 \mathrm{~B} 18$

398. B12+B18

$=>.9843750000000001 \mathrm{~B} 18+.0156249999999999 \mathrm{~B} 19$

399. B12+B19

$=>.9921875000000000 \mathrm{~B} 19+.00781250000000000 \mathrm{~B} 20$

400. $B 13+B 13=>B 14$

401. $\mathrm{B} 13+\mathrm{B} 14$

$=>.5000000000000000 \mathrm{~B} 14+.5000000000000000 \mathrm{~B} 15$

402. B13+B15

$=>.7499999999999999 B 15+.2500000000000001 B 16$

403. B13+B16

$=>.8749999999999999 B 16+.1250000000000001 \mathrm{~B} 17$

404. B13+B17

$=>.93750000000000000 \mathrm{~B} 17+.062500000000000000 \mathrm{~B} 18$

405. B13+B18

$=>.9687500000000000 \mathrm{~B} 18+.0312500000000000 \mathrm{~B} 19$

406. B13+B19

$=>.9843750000000001 B 19+.0156249999999999 B 20$

407. B14+B14=>B15

408. $\mathrm{B} 14+\mathrm{B} 15$

$=>.5000000000000000 \mathrm{~B} 15+.5000000000000000 \mathrm{~B} 16$

409. B14+B16

$=>.7499999999999999 \mathrm{~B} 16+.2500000000000001 \mathrm{~B} 17$

410. B14+B17

$=>.8749999999999999 \mathrm{~B} 17+.1250000000000001 \mathrm{~B} 18$

411. B14+B18

\begin{tabular}{|c|c|c|}
\hline $6.39 E+12$ & 0.5 & 0.0 \\
\hline $7.65 \mathrm{E}+12$ & 0.5 & $\odot . \odot$ \\
\hline $9.75 \mathrm{E}+12$ & 0.5 & 0.0 \\
\hline $1.30 \mathrm{E}+13$ & 0.5 & $\odot . \odot$ \\
\hline $1.81 \mathrm{E}+13$ & 0.5 & 0.0 \\
\hline $2.57 \mathrm{E}+13$ & 0.5 & 0.0 \\
\hline $3.74 \mathrm{E}+13$ & 0.5 & 0.0 \\
\hline $5.53 E+13$ & 0.5 & $\odot . \odot$ \\
\hline $8.28 \mathrm{E}+13$ & 0.5 & $\odot . \odot$ \\
\hline $6.49 \mathrm{E}+12$ & 0.5 & $\odot . \odot$ \\
\hline $7.17 \mathrm{E}+12$ & 0.5 & 0.0 \\
\hline $8.58 \mathrm{E}+12$ & 0.5 & 0.0 \\
\hline 1. $09 \mathrm{E}+13$ & 0.5 & 0.0 \\
\hline 1. $46 \mathrm{E}+13$ & 0.5 & $\odot . \odot$ \\
\hline $2.03 E+13$ & 0.5 & 0.0 \\
\hline $2.89 E+13$ & 0.5 & $\odot . \odot$ \\
\hline $4.20 \mathrm{E}+13$ & 0.5 & 0.0 \\
\hline $6.21 E+13$ & 0.5 & $\odot . \odot$ \\
\hline $7.28 \mathrm{E}+12$ & 0.5 & 0.0 \\
\hline $8.05 \mathrm{E}+12$ & 0.5 & $\odot . \odot$ \\
\hline $9.63 \mathrm{E}+12$ & $\odot .5$ & $\odot . \odot$ \\
\hline 1. $23 \mathrm{E}+13$ & 0.5 & 0.0 \\
\hline $1.64 \mathrm{E}+13$ & 0.5 & $\odot . \odot$ \\
\hline $2.28 \mathrm{E}+13$ & 0.5 & $\odot . \odot$ \\
\hline $3.24 \mathrm{E}+13$ & 0.5 & 0.0 \\
\hline $4.71 E+13$ & 0.5 & 0.0 \\
\hline $8.17 \mathrm{E}+12$ & 0.5 & 0.0 \\
\hline $9.04 \mathrm{E}+12$ & 0.5 & 0.0 \\
\hline 1. $08 \mathrm{E}+13$ & 0.5 & 0.0 \\
\hline $1.38 \mathrm{E}+13$ & 0.5 & 0.0 \\
\hline $1.85 \mathrm{E}+13$ & 0.5 & 0.0 \\
\hline $2.56 \mathrm{E}+13$ & 0.5 & $\odot . \odot$ \\
\hline $3.64 \mathrm{E}+13$ & 0.5 & 0.0 \\
\hline $9.17 \mathrm{E}+12$ & 0.5 & $\odot . \odot$ \\
\hline 1. $01 \mathrm{E}+13$ & 0.5 & $\odot . \odot$ \\
\hline 1. $21 \mathrm{E}+13$ & 0.5 & $\odot .0$ \\
\hline $1.55 \mathrm{E}+13$ & 0.5 & 0.0 \\
\hline 2. $07 \mathrm{E}+13$ & 0.5 & 0.0 \\
\hline
\end{tabular}




\begin{tabular}{|c|c|c|c|c|}
\hline 412 . & $\begin{array}{l}\text { B14+B19 } \\
=>.9687500000000000 B 19+.0312500000000000 B 20\end{array}$ & $2.87 \mathrm{E}+13$ & 0.5 & 0.0 \\
\hline 413. & $\mathrm{~B} 15+\mathrm{B} 15=>\mathrm{B} 16$ & $1.03 \mathrm{E}+13$ & 0.5 & 0.0 \\
\hline 414. & $\begin{array}{l}\text { B15+B16 } \\
=>.5000000000000000 \mathrm{~B} 16+.5000000000000000 \mathrm{~B} 17\end{array}$ & $1.14 \mathrm{E}+13$ & 0.5 & 0.0 \\
\hline 415. & $\begin{array}{l}\text { B15+B17 } \\
=>.7499999999999999 B 17+.2500000000000001 B 18\end{array}$ & $1.36 \mathrm{E}+13$ & 0.5 & $\odot . \odot$ \\
\hline 416. & $\begin{array}{l}\text { B15+B18 } \\
=>.8749999999999999 B 18+.1250000000000001 B 19\end{array}$ & $1.74 \mathrm{E}+13$ & 0.5 & 0.0 \\
\hline 417. & $\begin{array}{l}\text { B15+B19 } \\
=>.9375000000000000 \mathrm{~B} 19+.0625000000000000 \mathrm{~B} 20\end{array}$ & $2.32 \mathrm{E}+13$ & 0.5 & 0.0 \\
\hline 418. & $\mathrm{~B} 16+\mathrm{B} 16=>\mathrm{B} 17$ & $1.16 \mathrm{E}+13$ & 0.5 & 0.0 \\
\hline 419. & $\begin{array}{l}\text { B16+B17 } \\
=>.5000000000000000 B 17+.5000000000000000 B 18\end{array}$ & $1.28 \mathrm{E}+13$ & 0.5 & $\odot . \odot$ \\
\hline 420. & $\begin{array}{l}\text { B16+B18 } \\
=>.7499999999999999 B 18+.2500000000000001 B 19\end{array}$ & $1.53 \mathrm{E}+13$ & 0.5 & 0.0 \\
\hline 421. & $\begin{array}{l}\text { B16+B19 } \\
=>.8749999999999999 B 19+.1250000000000001 B 20\end{array}$ & $1.95 \mathrm{E}+13$ & 0.5 & 0.0 \\
\hline 422. & $\mathrm{~B} 17+\mathrm{B} 17=>\mathrm{B} 18$ & $1.30 \mathrm{E}+13$ & 0.5 & 0.0 \\
\hline 423. & $\begin{array}{l}\text { B17+B18 } \\
=>.5000000000000000 B 18+.5000000000000000 \mathrm{~B} 19\end{array}$ & $1.43 \mathrm{E}+13$ & 0.5 & 0.0 \\
\hline 424. & $\begin{array}{l}\text { B17+B19 } \\
=>.7499999999999999 B 19+.2500000000000001 B 20\end{array}$ & $1.72 \mathrm{E}+13$ & 0.5 & 0.0 \\
\hline 425. & $\mathrm{~B} 18+\mathrm{B} 18=>\mathrm{B} 19$ & $1.46 \mathrm{E}+13$ & 0.5 & 0.0 \\
\hline 426. & $\begin{array}{l}\text { B18+B19 } \\
=>.5000000000000000 \mathrm{~B} 19+.5000000000000000 \mathrm{~B} 20\end{array}$ & $1.61 \mathrm{E}+13$ & 0.5 & 0.0 \\
\hline 427. & $\mathrm{~B} 19+\mathrm{B} 19=>\mathrm{B} 20$ & $1.63 \mathrm{E}+13$ & 0.5 & 0.0 \\
\hline
\end{tabular}

